\title{
A simple and fast phase transition relaxation solver for compressible multicomponent two-phase flows
}

\author{
Alexandre Chiapolino ${ }^{\mathrm{a}, \mathrm{b}}$, Pierre Boivin ${ }^{1 \mathrm{a}}$, Richard Saurel ${ }^{\mathrm{a}, \mathrm{b}, \mathrm{c}}$ \\ ${ }^{a}$ Aix Marseille Univ, CNRS, Centrale Marseille, M2P2, Marseille, France \\ ${ }^{b}$ RS2N, Chemin de Gaumin, 83640 Saint-Zacharie, France \\ ${ }^{c}$ University Institute of France
}

\begin{abstract}
The present paper aims at building a fast and accurate phase transition solver dedicated to unsteady multiphase flow computations. In a previous contribution (Chiapolino et al. 2017), such a solver was successfully developed to compute thermodynamic equilibrium between a liquid phase and its corresponding vapor phase. The present work extends the solver's range of application by considering a multicomponent gas phase instead of pure vapor, a necessary improvement in most practical applications. The solver proves easy to implement compared to common iterative procedures, and allows systematic CPU savings over $50 \%$, at no cost in terms of accuracy. It is validated against solutions based on an accurate but expensive iterative solver. Its capability to deal with cavitating, evaporating and condensing two-phase flows is highlighted on severe test problems both 1D and 2D.
\end{abstract}

Keywords: multiphase flows, interfaces, hyperbolic systems, relaxation, phase transition.

\section{Introduction}

Phase transition between liquid and gas phases is a well-established phenomenon, which tends to relax thermochemical potentials toward equilibrium. Yet, finding the thermodynamic equilibrium can be tedious, as the corresponding equations are non-linear, the culprit being the non-trivial relation linking pressure $(p)$ and temperature $(T)$ at saturation. In multiphase compressible flows modeling, phase transition has dominant effects in many applications, and has to be accounted for via appropriate phase transition models. Iterative equilibrium solvers are

\footnotetext{
${ }^{1}$ Corresponding author: pierre.boivin@univ-amu.fr
} 
usually computationally expensive, as they require complex root-finding procedures, sometimes draining more CPU time than the flow computation itself. Their coding is not trivial, as for example, pure phase bounds require special attention.

Our previous work [1] presented a robust, fast and accurate alternative to the exact equilibrium solver in the case of flows consisting of a liquid phase in equilibrium with its own vapor. Speedup on the order of $50 \%$ was achieved on computations of such flows. When only liquid and the corresponding vapor are present, $p$ and $T$ are linked by the saturation curve $p=p_{\text {sat }}(T)$, simplifying somewhat the system to solve.

In the present paper, we aim at extending the model to liquid in equilibrium with multicomponent gases. In this case, $p$ and $T$ are not directly on the saturation curve, but are related through the partial pressure of the vapor component in the multicomponent gas phase. This allows the well-known existence of water vapor in air at atmospheric conditions, albeit a temperature below the boiling point.

The range of flow solvers to which the present model applies is identical to its previous version: it is designed in association with non-equilibrium hyperbolic flow models, such as Baer and Nunziato's (1986) [2] and its reduced versions. This includes the 5-equation model of Kapila et al. (2001) [3] and its extension for cavitating flows [4 6], as well as formulations for thermal equilibrium two-phase mixtures such as the Homogeneous Relaxation Model (HRM) and Homogeneous Equilibrium Model (HEM) [7, 8].

This paper is organized as follows. The first two sections remind the 4-equation flow model [6] and the corresponding thermodynamic closure, backbone of the present study. The 4-equation two-phase model is indeed the simplest flow model for the relaxation solver. Also, a wide range of applications can be addressed with this approach [6]. The third section presents the system of equations to be solved to relax thermochemical potentials, while the fourth details how to do so. The last sections are dedicated to displaying and validating the algorithm capabilities, through a series of 1D and 2D test cases involving cavitating, evaporating and condensing flows. 


\section{Flow model}

\subsection{Temperature-pressure equilibrium two-phase flow model}

The phase transition relaxation solver may be used with models mentioned previously (with 7, 5, 4 and 3 partial differential equations) but its presentation is simplified in the context of the 4-equation model (often called HRM). When dealing with more sophisticated formulations, as for example the 7-equation model, extra ingredients have to be presented, such as velocity, pressure and temperature relaxation solvers, as done for example in [9, 10]. Here, there is a single step that makes the connection from the 4-equation model (modeling mixtures out of thermodynamic equilibrium) and the 3-equation model (mixtures in full equilibrium). The 4-equation model for a two-phase mixture reads:

$$
\left\{\begin{aligned}
\frac{\partial \rho}{\partial t}+\operatorname{div}(\rho \mathbf{u}) & =0 \\
\frac{\partial \rho \mathbf{u}}{\partial t}+\operatorname{div}(\rho \mathbf{u} \otimes \mathbf{u}+p \underline{\underline{I}}) & =0 \\
\frac{\partial \rho E}{\partial t}+\operatorname{div}((\rho E+p) \mathbf{u}) & =0 \\
\frac{\partial \rho_{l} \alpha_{l}}{\partial t}+\operatorname{div}\left(\rho_{l} \alpha_{l} \mathbf{u}\right) & =0
\end{aligned}\right.
$$

where the liquid mass conservation equation can be written alternatively as,

$$
\frac{\partial \rho Y_{l}}{\partial t}+\operatorname{div}\left(\rho Y_{l} \mathbf{u}\right)=0
$$

Here $Y_{l, g}, \alpha_{l, g}, \rho_{l, g}$ denote respectively the mass fraction, the volume fraction and the material density of the liquid ( $l$ subscript) and gas ( $g$ subscript) phases. $\rho$ represents the mixture density, $\mathbf{u}$ represents the mixture center of mass velocity, $p$ denotes the mixture pressure and $E$ the mixture total energy $\left(E=e+u^{2} / 2\right)$. The mixture specific internal energy is defined as $e=Y_{l} e_{l}+Y_{g} e_{g}$. Mass transfer has been omitted in System (1.1) as it is addressed later.

\subsection{Extension to a multicomponent gas phase}

Considering multicomponent effects within the gas phase needs additional mass balance equations that can be written as,

$$
\frac{\partial \rho_{g} \alpha_{g} y_{k}}{\partial t}+\operatorname{div}\left(\rho_{g} \alpha_{g} y_{k} \mathbf{u}\right)=0
$$


where $y_{k}$ denotes the $k$-th gas component mass fraction within the gas phase. In the thermodynamic closure considered in this study, and detailed in the next section, the gas phase is assumed to obey the ideal gas equation of state.

As the molar volume is independent on the gas constituent, it is equivalent to consider that the constituents are ideally mixed within the gas phase (each occupying the whole gas phase volume), or assume that each component within the gas phase occupies its own separate volume. The equivalence between these two approaches is clarified in the next section. Under the latter assumption, all mass balance equations can be written as:

$$
\frac{\partial \rho Y_{k}}{\partial t}+\operatorname{div}\left(\rho Y_{k} \mathbf{u}\right)=0
$$

where the subscript $k$ refers to the various physical and chemical components. In the following, let us introduce the following convention:

$-k=1$ for the liquid,

$-k=2$ for the gas component corresponding to vapor of species 1 ,

$-k=3, \ldots, N$ for the remaining gas components, considered non-condensable in this work.

This notation for the species conservation equation is more convenient, as $Y_{k}$ is now the mass fraction for the $k$-th species in the entire mixture (containing both liquid and gas), so that the conservation equation for each species within the gas phase is of the same form as the liquid mass conservation equation.

The extended two-phase flow model consequently reads,

$$
\begin{cases}\frac{\partial \rho}{\partial t}+\operatorname{div}(\rho \mathbf{u}) & =0 \\ \frac{\partial \rho \mathbf{u}}{\partial t}+\operatorname{div}(\rho \mathbf{u} \otimes \mathbf{u}+p \underline{\underline{I}}) & =0 \\ \frac{\partial \rho E}{\partial t}+\operatorname{div}((\rho E+p) \mathbf{u}) & =0 \\ \frac{\partial \rho Y_{k}}{\partial t}+\operatorname{div}\left(\rho Y_{k} \mathbf{u}\right) & =0\end{cases}
$$

with 


$$
E=e+\frac{1}{2} \mathbf{u}^{2}, \quad e=\sum_{k=1}^{N} Y_{k} e_{k}
$$

System (1.4) is clearly reminiscent of the reactive (or multicomponent) Euler equations widely used in chemically reacting flows. However, the thermodynamic closure differs significantly from the one used in gas mixtures as examined hereafter.

\section{Thermodynamic closure}

This section presents the equations of state (EOS) used for each pure component as well as the mixture equation of state. Preliminarily, let us demonstrate the equivalence between the ideal gas mixture model that follows Dalton's law, and a gas phase within each constituent is assumed to occupy its own volume, in the specific context of temperature and pressure equilibrium.

\subsection{The gas phase is ideally mixed: Dalton's law}

Associated mixture rules are:

$$
\begin{cases}T=T_{k} & : \text { mixture in temperature equilibrium } \\ V=V_{k} & : \text { each chemical species occupies the entire volume } \\ p=\sum_{k=2}^{N} p_{\text {partial }, k} & : \text { the mixture pressure is the sum of partial pressures } \\ e=\sum_{k=2}^{N} Y_{k} e_{k} & : \text { mixture internal energy definition }\end{cases}
$$

The ideal gas law for a given species reads,

$$
p_{\text {partial }, k} V=n_{k} \hat{R} T
$$

and the gas mixture pressure reads,

$$
p V=\hat{R} T \sum_{k=2}^{N} n_{k}
$$

where $\hat{R}$ denotes the universal gas constant in molar units and $n_{k}$ is the number of moles. In this relation the temperature is deduced from the caloric equation of state for the mixture, 


$$
e=\hat{R} T \sum_{k=2}^{N} \frac{Y_{k} / W_{k}}{\gamma_{k}-1},
$$

where $W_{k}$ denotes the molar mass and $\gamma_{k}$ the polytropic coefficient of chemical species $k$. Consequently, the pressure reads,

$$
p=\frac{\frac{e}{V} \sum_{k=2}^{N} n_{k}}{\sum_{k=2}^{N} \frac{Y_{k} / W_{k}}{\gamma_{k}-1}}=\frac{e \sum_{k=2}^{N} \frac{n_{k} W_{k}}{V} \frac{1}{W_{k}}}{\sum_{k=2}^{N} \frac{Y_{k} / W_{k}}{\gamma_{k}-1}}
$$

finally,

$$
p=\frac{\rho e \sum_{k=2}^{N} \frac{Y_{k}}{W_{k}}}{\sum_{k=2}^{N} \frac{Y_{k} / W_{k}}{\gamma_{k}-1}} .
$$

2.2. Each gas constituent occupies its own volume in pressure and temperature equilibrium

We now consider another configuration with separate chemical species. Corresponding mixture rules are,

$$
\begin{cases}T=T_{k} & : \text { mixture in temperature equilibrium } \\ v=\sum_{k=2}^{N} Y_{k} v_{k} & : \text { the total specific volume is the sum of fluids' specific subvolumes } \\ p=p_{k} & : \text { pressure equilibrium among the chemical species } \\ e=\sum_{k=2}^{N} Y_{k} e_{k} & : \text { mixture internal energy definition }\end{cases}
$$

The ideal gas law for a given species now reads,

$$
p_{k} V_{k}=n_{k} \hat{R} T
$$

In mass units it becomes,

$$
p_{k}=\frac{n_{k} W_{k}}{V_{k}} \frac{\hat{R}}{W_{k}} T=\rho_{k} \frac{\hat{R}}{W_{k}} T .
$$

Consequently the specific volume of a given chemical species as a function of pressure and temperature reads,

$$
v_{k}=\frac{\hat{R}}{W_{k}} \frac{T}{p_{k}} .
$$


This result is inserted into the specific volume definition,

$$
v=\sum_{k=2}^{N} Y_{k} v_{k}
$$

yielding,

$$
\frac{p}{T}=\rho \sum_{k=2}^{N} Y_{k} \frac{\hat{R}}{W_{k}} .
$$

The mixture energy definition is now considered, resulting in the same relation as in Section 2.1:

$$
e=\hat{R} T \sum_{k=2}^{N} \frac{Y_{k} / W_{k}}{\gamma_{k}-1} .
$$

Eliminating the temperature by combining these two relations, the following result is obtained:

$$
p=\frac{\rho e \sum_{k=2}^{N} \frac{Y_{k}}{W_{k}}}{\sum_{k=2}^{N} \frac{Y_{k} / W_{k}}{\gamma_{k}-1}} .
$$

It is clear that both ideal mixtures models (Dalton's law Eq. (2.1) and separate chemical species Eq. (2.2)) are equivalent when the fluids are ideal gases evolving in both temperature and pressure equilibrium. This remark simplifies dramatically the building of the mixture EOS. Before addressing this topic, let us present the EOS for the liquid phase and its vapor.

\subsection{EOS for pure constituent}

In this frame, it is assumed that each gaseous constituent $(k=2, \ldots N)$ obeys the ideal gas equation of state. The EOS coefficients for the vapor (species $k=2$ ) must be carefully computed, in accordance with the liquid $(k=1)$ EOS, as to fit the phase diagram. The building of such an EOS has been addressed in Le Métayer et al. (2004) [11], on the basis of the Stiffened Gas (SG) EOS. In later developments, the same authors proposed the "Noble-Abel Stiffened Gas" (NASG) [12], which improves considerably the liquid specific volume accuracy by taking into account the repulsif molecular effects in addition to those already present in the SG EOS (agitation and attraction). The main formulas for the NASG EOS read for a given constituent 
$k=1, \ldots N$ (liquid, vapor, gas),

$$
\left\{\begin{array}{l}
p_{k}\left(v_{k}, e_{k}\right)=\left(\gamma_{k}-1\right) \frac{\left(e_{k}-q_{k}\right)}{v_{k}-b_{k}}-\gamma_{k} p_{\infty, k} \\
T_{k}\left(p_{k}, v_{k}\right)=\frac{\left(v_{k}-b_{k}\right)\left(p_{k}+p_{\infty, k}\right)}{C_{v, k}\left(\gamma_{k}-1\right)} \\
g_{k}\left(p_{k}, T_{k}\right)=\left(\gamma_{k} C_{v, k}-q_{k}^{\prime}\right) T_{k}-C_{v, k} T_{k} \ln \left[\frac{T_{k}^{\gamma_{k}}}{\left(p_{k}+p_{\infty, k}\right)^{\gamma_{k}-1}}\right]+b_{k} p_{k}+q_{k} \\
c_{k}\left(p_{k}, v_{k}\right)=v_{k} \sqrt{\frac{\gamma_{k} v_{k}\left(p_{k}+p_{\infty, k}\right)}{v_{k}-b_{k}}}
\end{array}\right.
$$

where the following parameters are needed for each phase: $\gamma_{k}, p_{\infty, k}, C_{v, k}, q_{k}, q_{k}^{\prime}$ and $b_{k}$. These parameters are constant coefficients characteristic of the thermodynamic properties of the fluid. Among them the coefficient $b$ represents the covolume of the fluid. $g_{k}$ denotes the phase Gibbs free energy of fluid $k, g_{k}=h_{k}-T s_{k}$ with $h_{k}$ and $s_{k}$ respectively the specific enthalpy and entropy. $c_{k}$ denotes the speed of sound of fluid $k$.

The following results can be adapted to the SG EOS framework by setting $b_{k}=0$. For all gaseous constituents $(k=2, \ldots N), b_{k}=0$ and $p_{\infty, k}=0$, and the above system reduces to the ideal gas EOS. Note that, for the $k$-th gaseous constituent, the pressure $p_{k}$ is based on the subvolume containing the constituent. It is therefore equal to the pressure of the whole gas phase, and not to the partial pressure of the constituent in the gas phase. In the following, it is explicitly stated when a partial pressure is introduced.

The same strategy as in [11] and [12] is repeated in order to find a formulation connecting the saturation pressure and temperature, by equating the liquid and the vapor chemical potentials $g_{1}=g_{2}$, leading to the following equation:

$$
\ln \left(p_{\text {sat }}+p_{\infty, 2}\right)=A+\frac{B+E p_{\text {sat }}}{T_{\text {sat }}}+C \ln \left(T_{\text {sat }}\right)+D \ln \left(p_{\text {sat }}+p_{\infty, 1}\right)
$$

where,

$$
\begin{gathered}
A=\frac{C_{p, 1}-C_{p, 2}+q_{2}^{\prime}-q_{1}^{\prime}}{C_{p, 2}-C_{v, 2}}, \quad B=\frac{q_{1}-q_{2}}{C_{p, 2}-C_{v, 2}}, \\
C=\frac{C_{p, 2}-C_{p, 1}}{C_{p, 2}-C_{v, 2}}, \quad D=\frac{C_{p, 1}-C_{v, 1}}{C_{p, 2}-C_{v, 2}}, \quad E=\frac{b_{1}-b_{2}}{C_{p, 2}-C_{v, 2}} .
\end{gathered}
$$


For liquid water and steam, the NASG fluid parameters, determined in the [273-500K] temperature range are given in Table 1, In the same table, the coefficients for air 2 are given (in the frame of the ideal gas assumption).

\begin{tabular}{|l|c|c|c|}
\hline Coefficients & Liquid water & Water vapor & air \\
\hline$C_{p}(\mathrm{~J} / \mathrm{kg} / \mathrm{K})$ & 4285 & 1401 & 1007 \\
\hline$C_{v}(\mathrm{~J} / \mathrm{kg} / \mathrm{K})$ & 3610 & 955 & 719 \\
\hline$\gamma$ & 1.19 & 1.47 & 1.4 \\
\hline$P_{\infty}(\mathrm{Pa})$ & $702810^{5}$ & 0 & 0 \\
\hline$q(\mathrm{~J} / \mathrm{kg})$ & -1177788 & 2077616 & 0 \\
\hline$q^{\prime}(\mathrm{J} / \mathrm{kg} / \mathrm{K})$ & 0 & 14317 & 0 \\
\hline$b\left(\mathrm{~m}^{3} / \mathrm{kg}\right)$ & $6.6110^{-4}$ & 0 & 0 \\
\hline$W(\mathrm{~g} / \mathrm{mol})$ & 18 & 18 & 29 \\
\hline
\end{tabular}

Table 1: Noble Abel Stiffened Gas (NASG) coefficients for water and air determined in the temperature range $[273-500 K]$

These parameters are used in the computational examples of the present paper. A comparison between the NASG EOS and experimental data is displayed in Fig 1, showing good agreement.

\subsection{Mixture equation of state}

Each constituent is assumed to follow the NASG EOS (reduced to the ideal gas EOS for gaseous constituents). However the mixture equation of state, based on mechanical and thermal equilibrium has yet to be built. Under the assumption of mechanical and thermal equilibrium, an inherent assumption to the 4-equation model, we have:

$$
\left\{\begin{array}{l}
T=T_{k} \quad \forall k \\
p=p_{k} \quad \forall k \\
v=\sum_{k=1}^{N}\left(Y_{k} v_{k}\right) \\
e=\sum_{k=1}^{N}\left(Y_{k} e_{k}\right)
\end{array}\right.
$$

\footnotetext{
${ }^{2}$ Note that we only consider mass transfer between liquid and vapor, so the reference energies of the other gas components have no importance. If other mass transfers are to be considered (between gas species), reference energies have to be set appropriately.
} 

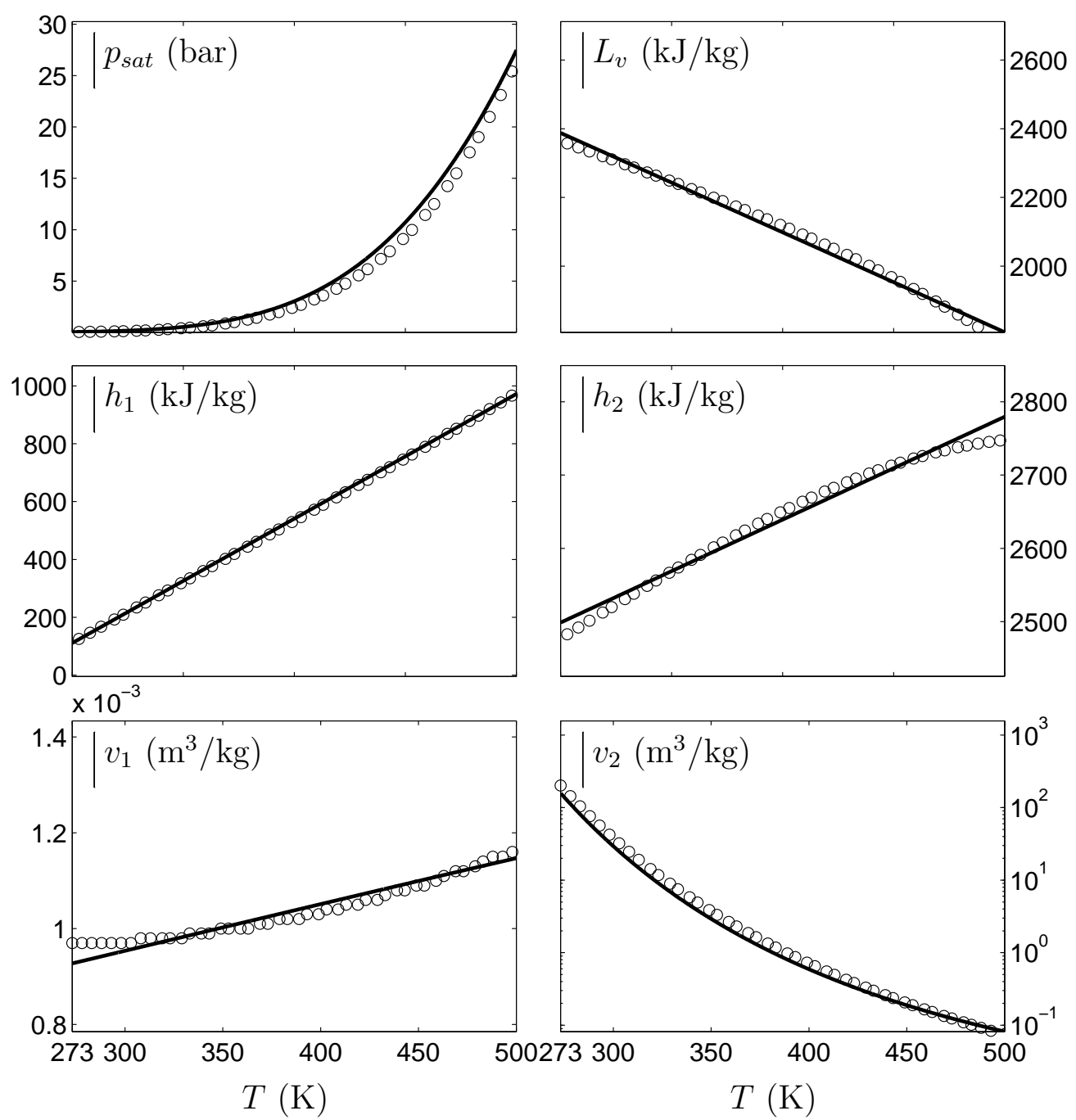

Figure 1: Comparison between experimental and theoretical saturation curves for liquid water and steam with coefficients determined in the temperature range $[273-500 \mathrm{~K}]$. The symbols represent the experimental data. The thick lines represent the NASG theoretical saturation curve. $P_{s a t}$ denotes the saturation pressure, $L_{v}$ the latent heat, $h$ the specific enthalpy and $v$ the specific volume. 
with $v$ the mixture specific volume.

As shown at the beginning of this section, considering (2.6) for the mixture of gases is equivalent to Dalton's law. Therefore the computed mixture pressure for the gas mixture is in agreement with both Dalton's law in the gas mixture and with the liquid-gas interface condition of equal pressures. Consequently, System (2.6) summarizes correctly the separate phase mixture model between the liquid and the gas mixture on one hand and the ideal gas mixture on the other hand.

From the expressions given in Eqs. (2.3) for pure constituents, the specific volumes and internal energies read:

$$
\left\{\begin{array}{l}
v_{k}\left(p_{k}, T_{k}\right)=\frac{\left(\gamma_{k}-1\right) C_{v, k} T_{k}}{p_{k}+p_{\infty, k}}+b_{k} \\
e_{k}\left(p_{k}, T_{k}\right)=\frac{p_{k}+\gamma_{k} p_{\infty, k}}{p_{k}+p_{\infty, k}} C_{v, k} T_{k}+q_{k},
\end{array}\right.
$$

which can be rewritten to give two expressions for the temperature ( since $T_{k}=T, \forall k$ ):

$$
\begin{aligned}
T & =\frac{v-\sum_{k=1}^{N} Y_{k} b_{k}}{\sum_{k=1}^{N} \frac{Y_{k}\left(\gamma_{k}-1\right) C_{v, k}}{p+p_{\infty, k}}}, \\
T & =\frac{e-\sum_{k=1}^{N} Y_{k} q_{k}}{\sum_{k=1}^{N} Y_{k} C_{v, k}\left(\frac{p+\gamma_{k} p_{\infty, k}}{p+p_{\infty, k}}\right)} .
\end{aligned}
$$

Equating these two expressions, and taking into account that $p_{\infty, k}=0$ and $b_{k}=0, \forall k>1$, a quadratic expression for the mixture pressure is obtained as:

$$
p=\frac{b+\sqrt{b^{2}+4 a c}}{2 a}
$$

with:

$$
\left\{\begin{array}{l}
a=\bar{C}_{v}, \\
b=\left(\frac{e-\bar{q}}{v-\bar{b}}\right)\left(\bar{C}_{p}-\bar{C}_{v}\right)-p_{\infty, 1} \bar{C}_{v}-p_{\infty, 1} Y_{1}\left(C_{p, 1}-C_{v, 1}\right), \\
c=\left(\frac{e-\bar{q}}{v-\bar{b}}\right) p_{\infty, 1}\left[\bar{C}_{p}-\bar{C}_{v}-Y_{1}\left(C_{p, 1}-C_{v, 1}\right)\right],
\end{array}\right.
$$

where mixture quantities are introduced:

$$
\bar{C}_{v}=\sum_{k=1}^{N} Y_{k} C_{v, k}, \quad \bar{C}_{p}=\sum_{k=1}^{N} Y_{k} C_{p, k}, \quad \bar{q}=\sum_{k=1}^{N} Y_{k} q_{k}, \quad \bar{b}=\sum_{k=1}^{N} Y_{k} b_{k} .
$$


Although not trivial, Eq. (2.10) ensures the strict positivity of the mixture pressure $p$ : if $Y_{1} \neq 1$, both $a$ and $c$ are strictly positive leading to $p>(b+|b|) / a$. The mixture pressure is then strictly positive. When $Y_{1}=1, a>0$ and $c=0$, and the strict positivity of the pressure is less trivial to demonstrate. However, combining Eqs. (2.7) and (2.11) in this limit leads to $b>0$, and consequently $p>0$, where $p$ follows the NASG EOS for the pure liquid phase. Let us add that when $Y_{1}=0$ (absence of liquid), the pressure equation (2.10) reduces to

$$
p=\left(\frac{e-\bar{q}}{v}\right) \frac{\left(\bar{C}_{p}-\bar{C}_{v}\right)}{\bar{C}_{v}},
$$

which after substituting $e=\sum_{k=2}^{N} Y_{k} e_{k}$, and noticing that $\left(\bar{C}_{p}-\bar{C}_{v}\right)=\sum_{k=2}^{N} Y_{k} \hat{R} / W_{k}$ for ideal gases, leads to:

$$
p=\sum_{k=2}^{N} Y_{k} \rho \hat{R} T / W_{k},
$$

or, in other words, the classical law of Dalton for the gas mixture. This provides an additional verification as to the possibility of considering each gas constituent to be in its own volume, as discussed in the flow model section. Albeit the apparent simplicity of the thermodynamic closure chosen, the phase transition model presented here may be extended to other thermodynamic closures provided each phase EOS is convex.

\section{Phase transition model}

\subsection{Objective}

When phase transition is addressed between the liquid and its vapor, only the conservation equations of the first two constituents are modified as:

$$
\left\{\begin{array}{l}
\frac{\partial \rho Y_{1}}{\partial t}+\operatorname{div}\left(\rho Y_{1} \mathbf{u}\right)=\rho \nu\left(g_{2}-g_{1}\right) \\
\frac{\partial \rho Y_{2}}{\partial t}+\operatorname{div}\left(\rho Y_{2} \mathbf{u}\right)=-\rho \nu\left(g_{2}-g_{1}\right)
\end{array}\right.
$$

where $g_{k}$ denotes the phase $k$ Gibbs free energy $g_{k}=h_{k}-T s_{k}$ with $h_{k}$ and $s_{k}$ respectively the specific enthalpy and entropy and $\nu\left(A_{I}, T, p\right)$ represents a relaxation parameter that controls the rate at which thermodynamic equilibrium is reached. It is a function of the specific interfacial area $A_{I}$, temperature and pressure. Its determination is possible only when the interfacial area 
$A_{I}$ is available, as with droplets and bubbly flows (see Furfaro and Saurel, (2016) for example [13]) and sometimes for stratified flows. A natural way to determine this exchange area would be to use a very high resolution mesh to capture interfaces at all spatial scales. However this strategy would require tremendous computing resources and can hardly be envisaged when considering large-sized problems. Nevertheless a realistic method in specific limit situations is to consider instantaneous thermodynamical relaxation between phases by the use of additional source terms [9]. In the present work, $\nu$ is considered very large, so that relaxation to thermodynamic equilibrium is immediate.

During the phase transition process, the mixture specific volume $v=1 / \rho$ and energy $e$ do not vary. The mass fractions for all gas species other than the vapor $\left(Y_{k \geq 3}\right)$ also remain constant. However, pressure and temperature do vary, reaching their equilibrium values $\left(p^{*}, T^{*}\right)$. The phase transition model thus reduces to computing the equilibrium state $\left(p^{*}, T^{*}, Y_{k}^{*}\right)$, at every time step, from the state described by $\left(p, T, v, e, Y_{k}\right)$.

Since pressure and temperature are functions of $\left(v, e, Y_{k}\right)$, and $v$ and $e$ are constant during the phase transition, the primary goal of the procedure is to compute accurately $Y_{1}^{*}$, since $Y_{2}^{*}$ is linked through mass conservation:

$$
Y_{2}^{*}=1-Y_{1}^{*}-\sum_{k \geq 3} Y_{k}
$$

In our approach, phase transition is decoupled of transport and wave propagation using a fractional step method, essentially reducing the model to setting $Y_{1}=Y_{1}^{*}$ and $Y_{2}=Y_{2}^{*}$ after each hyperbolic step (resolution of System (1.4) without mass transfer).

\subsection{Expression of the thermochemical equilibrium}

When only liquid and vapor are present $\left(Y_{k \geq 3}=0\right)$, as in our previous paper [1], the thermochemical equilibrium directly translates into $p^{*}=p_{\text {sat }}\left(T^{*}\right)$, independently of $Y_{1}^{*}$. Here an extra complexity is added, as the vapor partial pressure in the gas phase is equal to the saturation pressure at the current temperature.

The relation between $p^{*}$ and $T^{*}$ then depends on the composition of the multicomponent gas $Y_{k \geq 2}$. Within ideal gas mixtures, the vapor partial pressure is directly proportional to the vapor 
molar fraction:

$$
p_{\text {partial }}=\frac{Y_{2} / W_{2}}{\sum_{k \geq 2} Y_{k} / W_{k}} p,
$$

so that the expression satisfied at thermochemical equilibrium is

$$
p_{\text {sat }}\left(T^{*}\right)=p_{\text {partial }}=\frac{Y_{2}^{*} / W_{2}}{\sum_{k \geq 2} Y_{k}^{*} / W_{k}} p^{*} .
$$

This relation is indeed essential as it allows equilibrium of liquid and multicomponent gas in conditions below saturation (for instance, existence of water vapor at ambient temperature and pressure).

\subsection{Solution of a simplified problem}

Instead of solving thermochemical equilibrium at constant $v^{*}=v, e^{*}=e$ and $Y_{k \geq 3}^{*}=Y_{k \geq 3}$, and computing $\left(p^{*}, T^{*}, Y_{1}^{*}\right)$, let us assume $p^{*}=p, T^{*}=T$ and $Y_{k \geq 3}^{*}=Y_{k \geq 3}$ are constant, and $v$ and $e$ are varying. The thermochemical equilibrium problem is straightforward to solve analytically in these conditions:

Case $1 T>T_{\text {sat }}(p)$ : the mixture is necessarily purely gaseous. We then have $Y_{1}^{*}=0$.

Case $2 T<T_{\text {sat }}(p)$ : the mixture can be purely gaseous when the vapor quantity does not exceed its saturation limit, to be established hereafter. If this value is reached, then liquid is present.

The attainable limit for the vapor mass fraction before condensation in the multicomponent gas is obtained by solving $p_{\text {partial }}=p_{\text {sat }}(T)$. Using Eq. (3.2), one gets

$$
Y_{2}^{\text {sat }}=\frac{p_{\text {sat }}(T) W_{2}}{p-p_{\text {sat }}(T)} \sum_{k \geq 3} Y_{k} / W_{k} .
$$

Below the saturation temperature, we then have

$$
Y_{2}^{*}=\min \left(Y_{2}^{s a t}, 1-\sum_{k \geq 3} Y_{k}\right)
$$


The equilibrium mass fraction $Y_{2}^{*}$ cannot exceed the available room $\left(1-\sum_{k \geq 3} Y_{k}\right)$, nor can it exceed the saturation limit, after which liquid is present. $Y_{1}^{*}$ is then deduced from mass conservation. Figure 2 shows the solution for $Y_{1}^{*}$ and $Y_{2}^{*}$ of this simple problem, for a mixture of liquid water, water vapor and $\operatorname{air}(N=3)$, at atmospheric pressure, and a mixture temperature of $350 \mathrm{~K}$. On the right-hand side of the plot, there is so much air content that water vapor is diluted enough (below the saturation limit), and no liquid is present. On the left part, $Y_{2}^{*}$ varies linearly with $Y_{3}$, as a consequence of the saturation relation $p_{\text {partial }}=p_{\text {sat }}(T)$. The figure also indicates the variation of the mixture specific volume, which increases up to the saturation limit (since the liquid fraction diminishes), and then decreases, because air is heavier than water vapor.

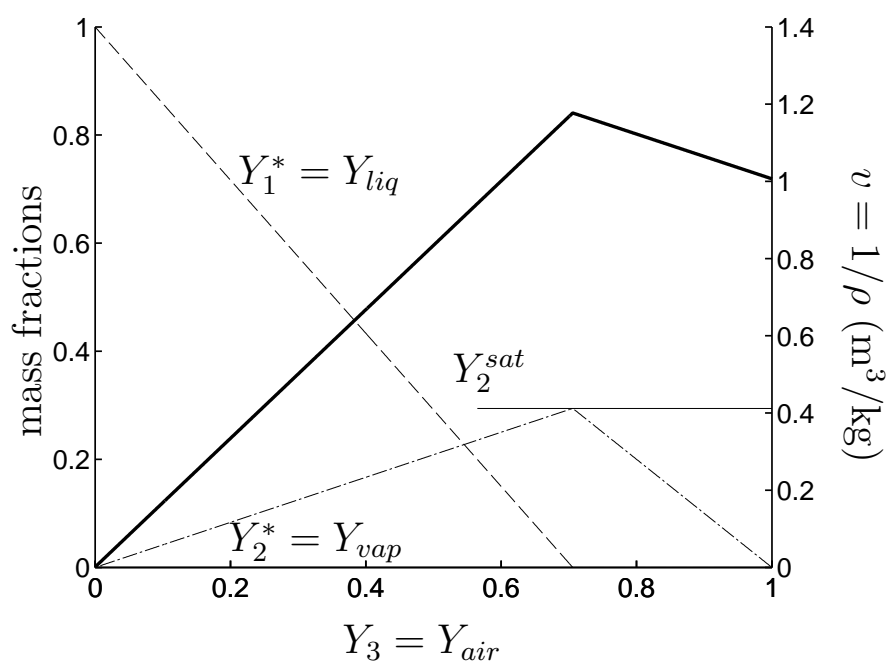

Figure 2: Equilibrium mass fractions obtained from water liquid/vapor, as a function of the air mass fraction. Conditions: atmospheric pressure, $\mathrm{T}=350 \mathrm{~K}$. dashed line: $Y_{1}^{*}$, dashed-dotted: $Y_{2}^{*}$. The thick line represents the specific volume obtained for the mixture in these conditions.

This simplified problem is convenient for two reasons: it illustrates well the problem to be solved, and also provides a simple way to compute initial conditions in a simulation.

When the problem is to be solved at constant $(v, e)$, instead of $(p, T)$, an analytic approach is no longer possible, and the problem has to be solved numerically. In the following section, we present an alternative to a tedious iterative process. 


\section{Thermochemical relaxation algorithm}

The idea of our method is to gradually reach the exact solution (typically in 1 to 3 time steps of the flow solver), by providing a fair approximation for $Y_{1}^{*}$, while iterative approaches, such as

the one promoted in Le Métayer et al. (2013) [9], directly computes the exact solution. In that direction, we will follow a similar strategy than in our previous work [1], extended to the fact that the relation between pressure and saturation pressure is modified compared to (3.3), and now depends on the result (since the partial pressure is a function of $Y_{2}$ ). First, let us bound the equilibrium mass fractions $Y_{1}^{*}$ and $Y_{2}^{*}$ :

$$
\left\{\begin{array}{l}
Y_{\min }=\epsilon \\
Y_{\max }=1-Y_{\min }-\sum_{k=3}^{N} Y_{k} .
\end{array}\right.
$$

With $\epsilon \rightarrow 0$ for numerical purposes, typically on the order of $10^{-8}$. As in our previous work [1], we first assume $Y_{1}^{*}=Y_{m i n}$, to check if the system has a solution without liquid. Under this assumption, pressure and temperature are computed by use of the mixture equation of state (2.8) and (2.10):

$$
\left\{\begin{array}{l}
p=p(v, e, Y), \\
T=T(v, e, Y),
\end{array}\right.
$$

since the mixture variables $v$ and $e$ are invariant through phase transition. Additionally, we compute the corresponding partial pressure for vapor (3.2), and compare it to the saturation pressure (2.4). If the partial pressure is below the saturation pressure, no liquid is present and the solution is $Y_{1}^{*}=Y_{\min }$ and $Y_{2}^{*}=Y_{\max }$. Note that, unlike in our last contribution [1], there is no need to check the existence of the pure liquid phase: no matter how small $\epsilon$ is chosen, there will always be a (very small) solution for $Y_{2}^{*}$ satisfying that its partial pressure is equal to the saturation pressure. Section 5 (about the algorithm's stability) provides additional comments 
and clarify this last feature. If $Y_{1}^{*} \neq Y_{\text {min }}$, the following system has to be solved

$$
\left\{\begin{array}{l}
p_{\text {partial }}=x_{v}^{*} \cdot p^{*}=p_{\text {sat }}\left(T^{*}\right), \\
v=Y_{1}^{*} v_{1}\left(T^{*}, p^{*}\right)+Y_{2}^{*} v_{2}\left(T^{*}, p^{*}\right)+\sum_{k=3}^{N} Y_{k} v_{k}\left(T^{*}, p^{*}\right), \\
e=Y_{1}^{*} e_{1}\left(T^{*}, p^{*}\right)+Y_{2}^{*} e_{2}\left(T^{*}, p^{*}\right)+\sum_{k=3}^{N} Y_{k} e_{k}\left(T^{*}, p^{*}\right),
\end{array}\right.
$$

leaving us with three equations for three unknowns $\left(p^{*}, T^{*}, Y_{1}^{*}\right)$. The vapor molar fraction $x_{v}^{*}$ is defined as,

$$
x_{v}^{*}=\frac{Y_{2}^{*} / W_{2}}{Y_{2}^{*} / W_{2}+\sum_{k=3}^{N} Y_{k} / W_{k}} .
$$

We will now evaluate three approximate expressions for $Y_{1}^{*}$. Rewriting the specific volume definition from System (4.2) leads to the following approximate expression:

$Y_{1}^{m}(p, T)=\frac{v-v_{g}(p, T)}{v_{1}(p, T)-v_{2}(p, T)}, \quad$ with $\quad v_{g}(p, T)=\left(1-\sum_{k=3}^{N} Y_{k}\right) v_{2}(p, T)+\sum_{k=3}^{N} Y_{k} v_{k}(p, T)$.

Any mass fraction computed from this formula after the hyperbolic step will satisfy the mass conservation $\forall(p, T)$. Similarly, we can rewrite the specific mixture energy definition from System (4.2):

$Y_{1}^{e}(p, T)=\frac{e-e_{g}(p, T)}{e_{1}(p, T)-e_{2}(p, T)}, \quad$ with $\quad e_{g}(p, T)=\left(1-\sum_{k=3}^{N} Y_{k}\right) e_{2}(p, T)+\sum_{k=3}^{N} Y_{k} e_{k}(p, T)$

Equations for $Y_{2}^{m}(p, T)$ and $Y_{2}^{e}(p, T)$ (expressed for the vapor phase instead of the liquid phase) can be obtained as,

$$
Y_{2}=1-Y_{1}-\sum_{k=3}^{N} Y_{k}
$$

In our previous work [1], these two functions of $(p, T)$ could be reduced to functions of $p$ in finding the equilibrium as $p=p_{\text {sat }}(T)$ for a liquid-vapor mixture. As shown in Eq. (3.3), the new relation $p_{\text {partial }}=p_{\text {sat }}\left(T^{*}\right)$ includes an additional dependence on $Y_{2}^{*}$ (or, equivalently, on 
$Y_{1}^{*}$, leading to Eq. (3.3), reminded here:

$$
Y_{2}^{\text {sat }}(p, T)=\frac{p_{\text {sat }}(T) W_{2}}{p-p_{\text {sat }}(T)} \sum_{k \geq 3} Y_{k} / W_{k} .
$$

The exact solution to the problem is found in pressure and temperature conditions satisfying all three Eqs. of (4.2), or, equivalently:

$$
Y_{2}^{m}\left(p^{*}, T^{*}\right)=Y_{2}^{e}\left(p^{*}, T^{*}\right)=Y_{2}^{s a t}\left(p^{*}, T^{*}\right)
$$

This is illustrated in Fig. 3, for a set of conditions initially out of equilibrium (30K below saturation).

In this graph,

- the light gray surface represents the mass conservation condition: $Y_{2}^{m}(p, T)$,

- the medium gray surface represents the energy conservation condition: $Y_{2}^{e}(p, T)$,

- the dark gray surface, represents the thermochemical equilibrium condition: $Y_{2}^{\text {sat }}(p, T)$.

The algorithm consists in computing three approximations for $Y_{2}^{*}$ :

$-Y^{m}=Y_{2}^{m}(p, T)$ is evaluated for the initial values of $\left(p, x_{v}\right)$ (from the hyperbolic step), and $T=T_{\text {sat }}\left(x_{v} \cdot p\right)$,

- $Y^{e}=Y_{2}^{e}(p, T)$ is evaluated for the initial values of $\left(p, x_{v}\right)$ (from the hyperbolic step), and $T=T_{\text {sat }}\left(x_{v} \cdot p\right)$,

- $Y^{\text {sat }}=Y_{2}^{\text {sat }}(p, T)$ is evaluated at the initial $(p, T)$.

As in [1], the idea of this method is then to pick the value with the smallest variation, under the condition that all three associated mass transfers are of the same sign. This idea is reminiscent of the well known Minmod slope limiter widely used in high order CFD solvers for gradients limitation. In other words, we introduce:

$$
\left\{\begin{array}{l}
r_{1}=\left(Y^{m}-Y_{2}\right)\left(Y^{e}-Y_{2}\right), \\
r_{2}=\left(Y^{m}-Y_{2}\right)\left(Y^{s a t}-Y_{2}\right),
\end{array}\right.
$$




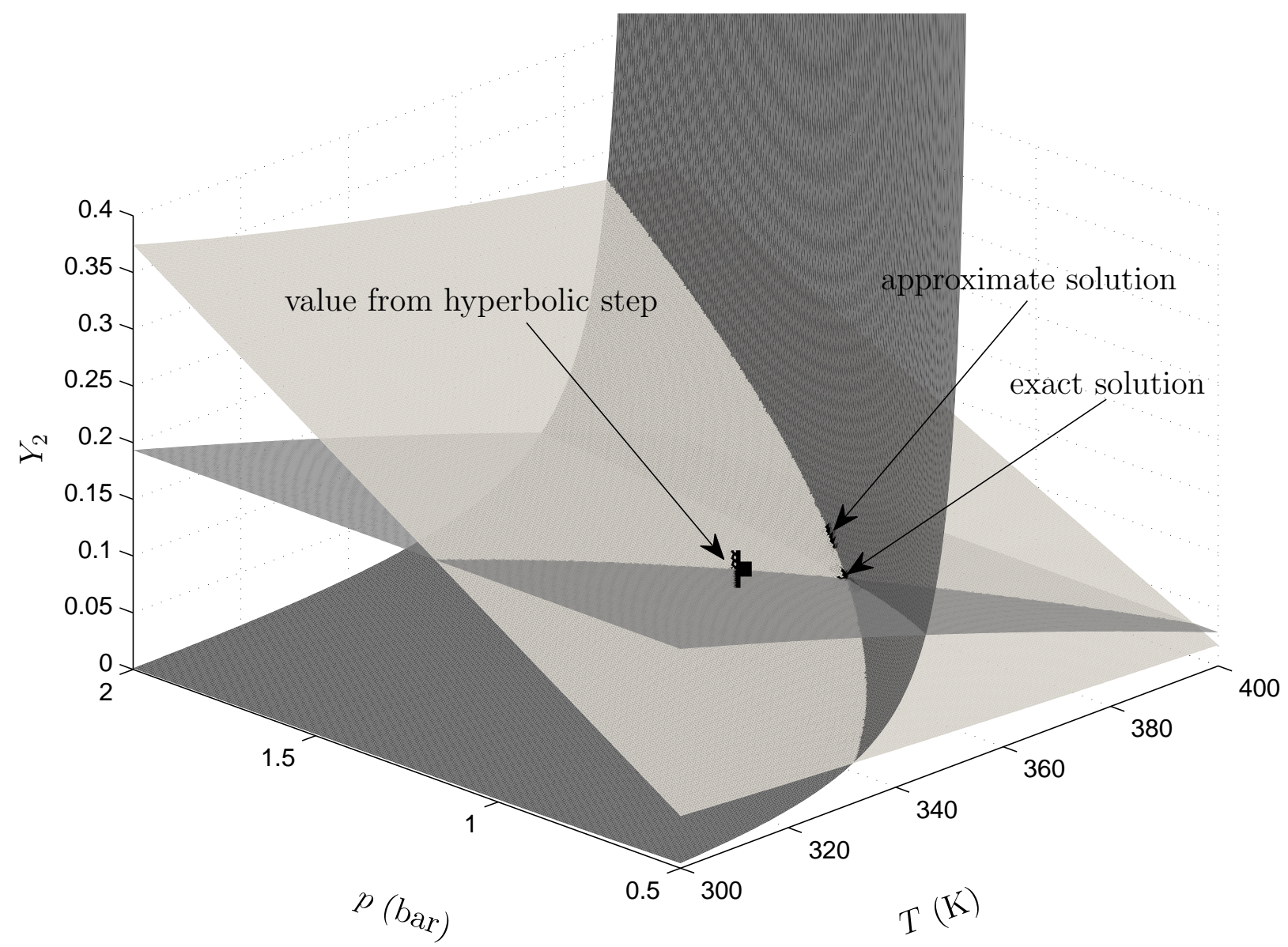

Figure 3: Illustration of the algorithm for the multicomponent phase transition solver. From light gray to dark gray: $Y^{m}\left(p^{*}, T^{*}\right), Y^{e}\left(p^{*}, T^{*}\right), Y^{\text {sat }}\left(p^{*}, T^{*}\right)$. The exact solution of the problem is found at the intersection of the three surfaces. The value from hyperbolic step corresponds to the initial conditions of the algorithm: $Y_{2}=2 . Y_{2}^{*}$ (far from equilibrium) at $p=1 \mathrm{~atm}, T=T_{\text {sat }}(p)-30 \mathrm{~K}=343 \mathrm{~K}$, and $Y_{3}=0.1$. Here, computations lead to $Y_{2}^{*}=0.1, Y^{m}=0.11, Y^{e}=0.9, Y^{\text {sat }}=0.03$, so that the algorithm retains $Y^{m}$, within $10 \%$ of the exact solution. 
where $Y_{2}$ is the initial mass fraction (from the hyperbolic step), and $Y^{m}, Y^{e}, Y^{\text {sat }}$ are three estimates for the equilibrium $Y_{2}^{*}$.

- If $r_{1}<0$, or $r_{2}<0$, no mass transfer happens: $Y_{2}^{*}=Y_{2}$

- Else, the minimum mass transfer is used. For instance, if $Y^{m}$ is the closest value to $Y_{2}$ (out of $Y^{m}, Y^{e}, Y^{s a t}$ ), then $Y_{2}^{*}=Y^{m}$.

Under the latter condition, it can be implemented as:

$$
Y_{2}^{*}=Y_{2}+\operatorname{sgn}\left[Y^{m}-Y_{2}\right] \times \operatorname{Min}\left[\left|Y^{m}-Y_{2}\right|,\left|Y^{e}-Y_{2}\right|,\left|Y^{s a t}-Y_{2}\right|\right]
$$

\section{Summary of the procedure:}

\section{1) Search for a solution without liquid}

- The limit case $Y_{l}^{*} \rightarrow \epsilon$ is considered with computation of the pressure and temperature according to the mixture equation of state (2.8) and (2.10). Additionally the corresponding saturation pressure and partial pressure for vapor are computed according to Eqs. (2.4) and (3.2).

- If the partial pressure is below the saturation pressure, no liquid is to be considered and the solution is $Y_{1}^{*}=Y_{\min }$ and $Y_{2}=Y_{\max }$ according to Eq. (4.1).

\section{2) If liquid is present, thermodynamic equilibrium is to be computed}

- Computation of the liquid mass fraction at equilibrium $Y_{1}^{*}$ is done according to the Minmod-like procedure Eq. (4.8) using Eqs. $Y^{m}$ (4.4), $Y^{e}$ (4.5), $Y^{\text {sat }}$ (4.6), (4.7), and the EOS (2.7).

\section{Algorithm's stability}

In a previous contribution [1] in which only liquid and vapor were considered, a major challenge was to deal with the pure liquid limit. Let us consider pure liquid water, initially at $300 \mathrm{~K}$ and $1 \mathrm{~atm}$. If a small quantity $\epsilon$ of vapor appears, the system has to go to thermochemical equilibrium, no matter how far off the initial conditions are. Because of the high heat capacity and low compressibility of liquid water, the process is mostly isothermal, and important pressure 
fluctuations could be observed. This motivated the introduction of a correction on the pressure used to estimate $Y^{m}$ and $Y^{e}$, in order to improve the method capabilities to deal with the transition from pure liquid to two-phase mixture in the limit $Y_{k \geq 2} \rightarrow 0$.

Here, there is no need for such a correction. The situation detailed above, in which a mixture can be found very far off equilibrium after the hyperbolic step, can not be encountered. In the present work's context, we can find a value for the vapor mass fraction $Y_{2}=Y_{v a p}$, no matter the conditions given, as traces of incondensable gas are present $Y_{3}=Y_{\text {air }}$. At $300 \mathrm{~K}$ and $1 \mathrm{~atm}$ for a mixture of liquid water, vapor water and air, assuming $Y_{3}=\epsilon$ (which can be as small as numerically allowed) will lead to a very small but non zero $Y_{2}$. With these considerations, the lack of continuity in the transition from pure liquid to two-phase mixture, present in the liquid-vapor model, vanishes in the present context.

Additionally, let us note that presence of air in water is physical (dissolved), and can be estimated through Henry's law. For instance, in ambient conditions, the air mass fraction dissolved is on the order of $Y_{3}=10^{-5}$, leading to a value of $Y_{2}$ of the same order, satisfying System (4.2). Indeed, such low values do not have significant effect on mean density and energy, which remain close to the pure phase values.

In practical computations presented in this paper, unless stated otherwise, a small value is set for $Y_{3}$ in the "pure" liquid, and $Y_{2}$ is computed accordingly, following Eq. (3.5) for the initial prescribed pressure and temperature.

Another key property responsible for the algorithm's stability is that $\forall(p, T)$ (a priori away from the equilibrium point), the exact solution for $Y_{2}^{*}$ always lies between the minimum and maximum values of $Y_{2}^{m}(p, T), Y_{2}^{e}(p, T), Y_{2}^{s a t}(p, T)$ This is clearly visible in Fig. 3, Although we have not proved this result because of the non-linear dependence of $p_{\text {sat }}(T)$ (as in our previous contribution), we have not found a case in which this does not apply. As a consequence:

- If $Y_{2}>Y_{2}^{*}$ initially, mass transfer will be activated only if $Y^{m}, Y^{e}$ and $Y^{\text {sat }}$ are all smaller than $Y_{2}$. Given the above property, one of them at least is superior to $Y_{2}^{*}$. The algorithm then cannot "overestimate" the mass transfer.

- The same thing can be said when $Y_{2}<Y_{2}^{*}$ initially. 
- Last but not least, since $0<Y_{2}<1-\sum_{k \geq 3} Y_{k}$ (given the hyperbolic step is properly implemented), and the exact solution also satisfies $0<Y_{2}^{*}<1-\sum_{k \geq 3} Y_{k}$, the approximate solution from the algorithm can never go out of bounds.

\section{Numerical results}

Robustness and accuracy of the present thermochemical relaxation algorithm have been illustrated in a previous contribution [1] for two-phase flows containing a liquid and its own vapor only. In the current context, non-condensable gases are present. The proposed relaxation solver is to be compared to an iterative algorithm such as the one promoted in Le Métayer et al. (2013) [9]. Unlike this last algorithm, the notion of vapor molar fraction and partial pressures in the gas phase must be taken into account, bringing additional difficulties. The corresponding iterative solver is consequently recalled and modified in Appendix.

To illustrate robustness and accuracy of the relaxation algorithm, a shock tube containing liquid water, its own vapor and some non-condensable air is considered, with variable initial conditions. Shock tube tests appear as excellent benchmarks as the flow contains shock waves, contact discontinuities and rarefaction fans that create some arduous conditions. In this section, the tube is 1 meter long and the initial discontinuity is located at 0.5 meter. Boundary conditions are non-reflecting ones. The computations that follow are addressed with the MUSCL Hancock method using van Leer's slope limiter and the HLLC Riemann solver (see Toro (1997) [14], Saurel et al. (2016) [6] for details). This section compares the results obtained with the present relaxation solver and the classic root-finding procedure method given in Appendix. Unless stated otherwise, the one-dimensional simulations are carried out on meshes with 100 cells.

\subsection{Shock tube test with a mixture far from the phase bounds}

A two-phase mixture with initial mass fractions set to $Y_{1}=0.1$ (liquid), $Y_{2}=0.2$ (vapor) and $Y_{3}=0.7$ (air) is considered throughout the entire tube with an initial pressure ratio of 2 , resulting in the presence of initial density and temperature discontinuities.

In order to find an initial thermodynamic equilibrium, the temperatures of both chambers 
are deduced as

$$
T=T_{\text {sat }}\left(x_{v} p\right) \quad \text { with } \quad x_{v}=\frac{Y_{2} / W_{2}}{Y_{2} / W_{2}+\sum_{k=3}^{N} Y_{k} / W_{k}} .
$$

The mixture density is then computed as,

$$
\rho=\frac{1}{v} \quad \text { with } \quad v=\sum_{k=1}^{N} Y_{k} v_{k}(p, T),
$$

where $v_{k}(p, T)$ is given by Eq. (2.7). The results are shown at time $t \approx 1 \mathrm{~ms}$ in Fig. 4 .
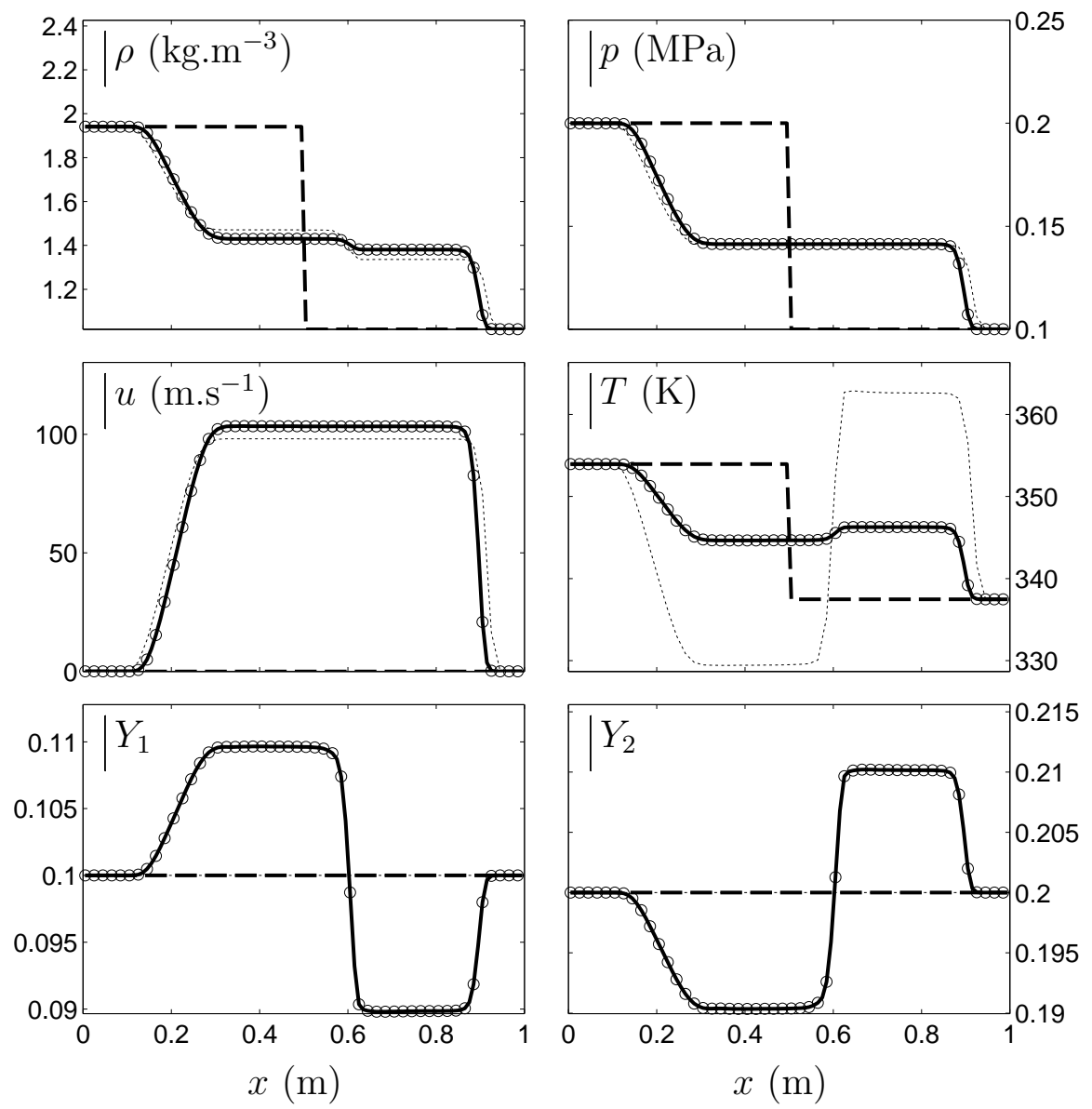

Figure 4: Comparison of the present relaxation algorithm (thick lines) versus the non-linear iterative solver (symbols). Shock tube test with a two-phase mixture made of liquid water, vapor water and air. The dotted lines represent the solutions without phase transition. The dashed lines represent the initial conditions: $p_{l}=2 \cdot 10^{5}$ $\mathrm{Pa}, p_{r}=10^{5} \mathrm{~Pa}, u_{l}=u_{r}=0 \mathrm{~m} . \mathrm{s}^{-1}, Y_{1}^{\text {left }}=Y_{1}^{\text {right }}=0.1, Y_{2}^{\text {left }}=Y_{2}^{\text {right }}=0.2$ and $Y_{3}^{\text {left }}=Y_{3}^{\text {right }}=0.7$. Final time: $t \approx 1$ ms. Mesh: 100 cells. For the sake of clarity, only 50 symbols out of 100 are plotted for the iterative method. 
Perfect agreement is obtained between the present relaxation solver and the non-linear iterative method. The shock compression yields evaporation and the expansion wave results in condensation in this example.

\subsection{Shock tube test with a mixture with air in major proportions}

Mass fractions of the previous test were given as inputs and the temperatures were deduced in order to respect initial thermodynamic equilibrium. Let us now consider a different point of view. As it is easier to measure or impose temperature in a given flow, it is now used as initial input. Pressures, velocities and the mass fraction of the non-condensable gas (air) remain inputs as well. Proportions of liquid and vapor are then deduced according to Eq. (3.5).

The following test considers a shock tube with an initial pressure ratio of 2 and initial temperature of $T=293 \mathrm{~K}$ throughout the tube. The air mass fraction is initially set to $Y_{3}=0.98$ in the whole tube. The liquid and vapor mass fractions are then deduced as $Y_{1} \simeq 1.073 \cdot 10^{-2}$ and $Y_{2} \simeq 9.263 \cdot 10^{-3}$ in the left chamber and $Y_{1} \simeq 1.186 \cdot 10^{-3}$ and $Y_{2} \simeq 1.881 \cdot 10^{-2}$ in the right chamber. The results are shown at time $t \approx 1 \mathrm{~ms}$ in Fig. 5 ,

Perfect agreement is obtained between the two solvers. The shock compression results in total evaporation of the liquid water and the rarefaction results in condensation. Disappearance of liquid water is computed without oscillations by the two methods.

\subsection{Shock tube test with a mixture mainly made of water}

Mass fractions from the previous test case are reversed by considering $Y_{3}=10^{-5}$ and $T=293$ K initially throughout the tube, corresponding to a subcooled liquid. The following test considers a shock tube with an initial pressure ratio of 2 . The liquid and vapor mass fractions are then deduced as $Y_{1} \simeq 0.9999899$ and $Y_{2} \simeq 9.426 \cdot 10^{-8}$ in the left chamber and $Y_{1} \simeq 0.99989$ and $Y_{2} \simeq 1.919 \cdot 10^{-7}$ in the right chamber. The results are shown at time $t \approx 1.5 \mathrm{~ms}$ in Fig. 6 ,

Perfect agreement is again obtained between the two solvers. The liquid phase having the highest internal heat capacity, the resulting flow is quasi-isothermal despite the slight evaporation and condensation processes appearing through the shock and rarefaction. 

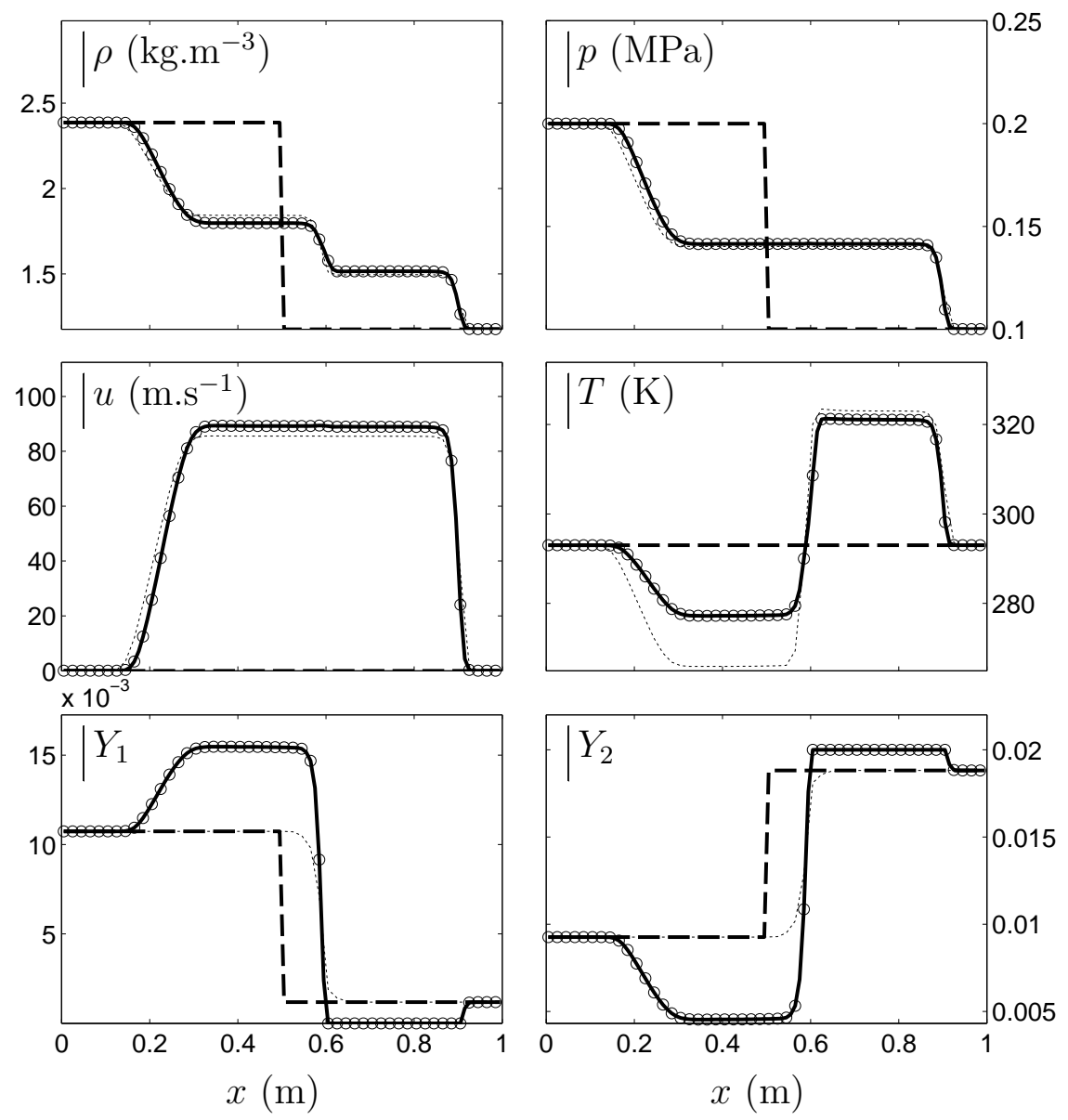

Figure 5: Comparison of the present relaxation algorithm (thick lines) versus the non-linear iterative solver (symbols). Shock tube test with a two-phase mixture with air in major proportions. The dotted lines represent the solutions without phase transition. The dashed lines represent the initial conditions: $p_{l}=2 \cdot 10^{5} \mathrm{~Pa}, p_{r}=10^{5}$ $\mathrm{Pa}, u_{l}=u_{r}=0 \mathrm{~m} \cdot \mathrm{s}^{-1}, Y_{1}^{\text {left }} \simeq 1.073 \cdot 10^{-2}, Y_{1}^{\text {right }} \simeq 1.186 \cdot 10^{-3}, Y_{2}^{\text {left }} \simeq 9.263 \cdot 10^{-3}, Y_{2}^{\text {right }} \simeq 1.881 \cdot 10^{-2}$ and $Y_{3}^{\text {left }}=Y_{3}^{\text {right }}=0.98$. Final time: $t \approx 1 \mathrm{~ms}$. Mesh: 100 cells. For the sake of clarity, only 50 symbols out of 100 are plotted for the iterative method. Full liquid evaporation is correctly computed by both methods. 

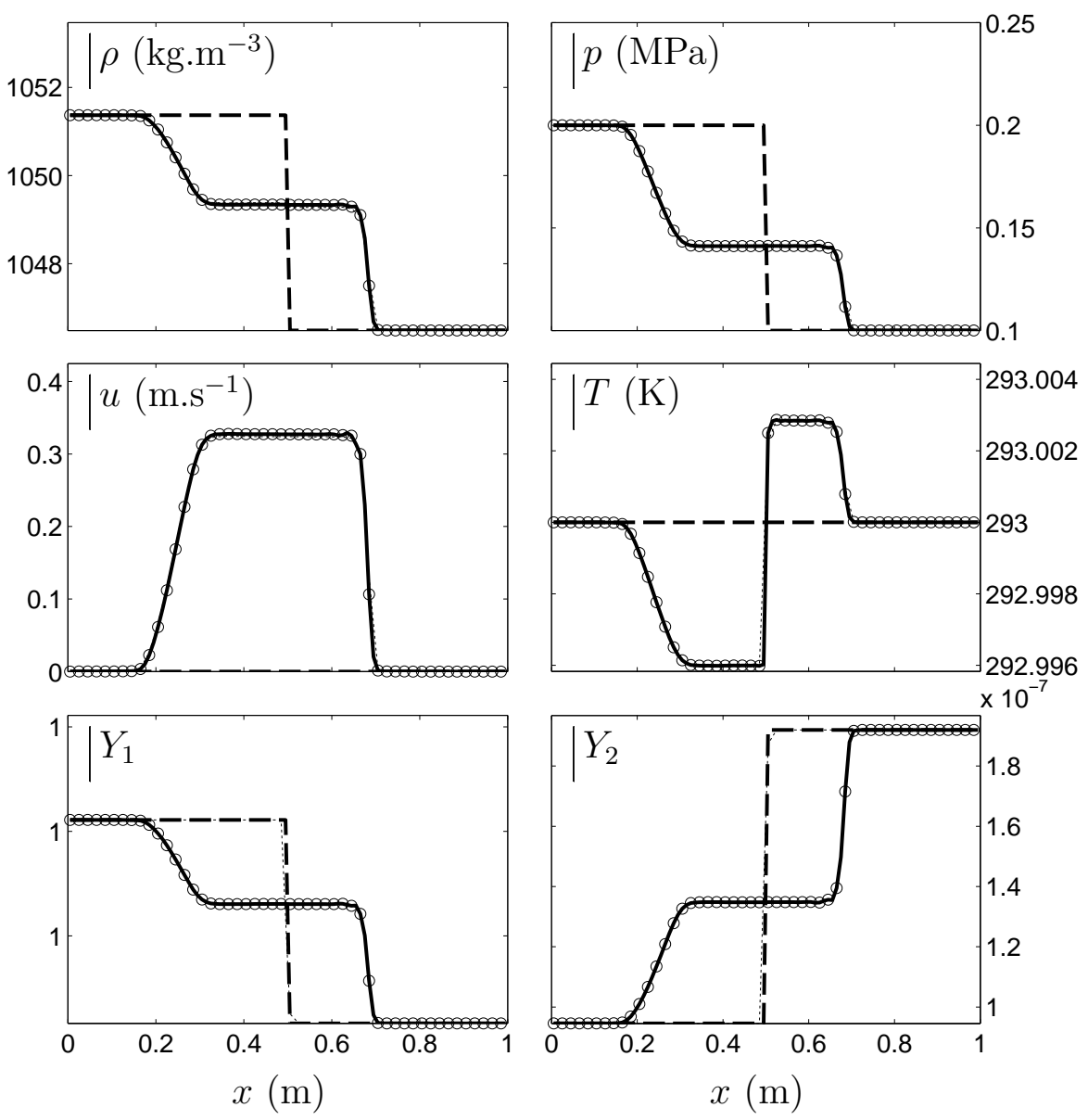

Figure 6: Comparison of the present relaxation algorithm (thick lines) versus the non-linear iterative solver (symbols). Shock tube test with a two-phase mixture mainly made of liquid water. The dotted lines represent the solutions without phase transition. The dashed lines represent the initial conditions: $p_{l}=2 \cdot 10^{5} \mathrm{~Pa}, p_{r}=10^{5}$ $\mathrm{Pa}, u_{l}=u_{r}=0 \mathrm{~m} \cdot \mathrm{s}^{-1}, Y_{1}^{\text {left }} \simeq 0.9999899, Y_{1}^{\text {right }} \simeq 0.99989, Y_{2}^{\text {left }} \simeq 9.45 \cdot 10^{-8}, Y_{2}^{\text {right }} \simeq 1.919 \cdot 10^{-7}$ and $Y_{3}^{\text {left }}=Y_{3}^{\text {right }}=10^{-5}$. Final time: $t \approx 1.5 \mathrm{~ms}$. Mesh: 100 cells. For the sake of clarity, only 50 symbols out of 100 are plotted for the iterative method. 


\subsection{Double expansion test with a two-phase mixture mainly made of liquid water}

The following test mimics cavitation in an initial subcooled liquid. The initial pressure, temperature and air mass fractions are respectively 1 bar, $T=293 \mathrm{~K}$ and $Y_{3}=10^{-5}$ throughout the entire tube. The liquid and vapor mass fractions are then deduced as $Y_{1} \simeq 0.99989$ and $Y_{2} \simeq 1.919 \cdot 10^{-7}$ in the left and right chambers. The initial velocity is set to $-1 \mathrm{~m} \cdot \mathrm{s}^{-1}$ at left and $+1 \mathrm{~m} \cdot \mathrm{s}^{-1}$ at right. The results are shown at time $t \approx 3.5 \mathrm{~ms}$ in Fig. 7 .
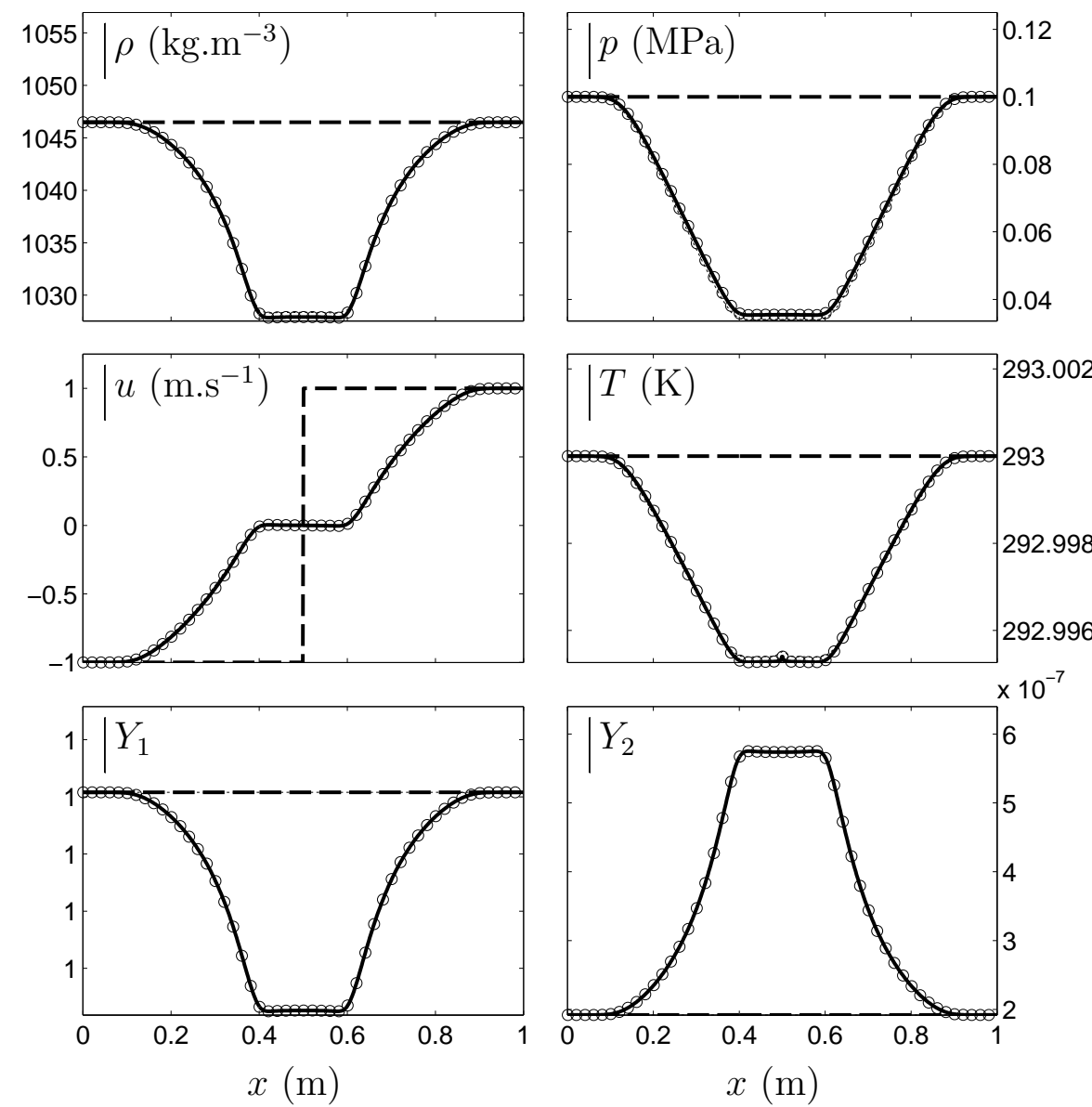

Figure 7: Comparison of the present relaxation algorithm (thick lines) versus the non-linear iterative solver (symbols). Double expansion test with a mixture mainly made of liquid water. The dotted lines represent the solutions without phase transition. The dashed lines represent the initial conditions: $p_{l}=2 \cdot 10^{5} \mathrm{~Pa}$, $p_{r}=10^{5} \mathrm{~Pa}, u_{l}=-1 \mathrm{~m} . \mathrm{s}^{-1}, u_{r}=+1 \mathrm{~m} . \mathrm{s}^{-1}, Y_{1}^{\text {left }}=Y_{1}^{\text {right }} \simeq 0.99989, Y_{2}^{\text {right }}=Y_{2}^{\text {right }} \simeq 1.919 \cdot 10^{-7}$ and $Y_{l}^{\text {air }}=Y_{r}^{\text {air }}=10^{-4}$. Final time: $t \approx 3.5 \mathrm{~ms}$. Mesh: 100 cells. For the sake of clarity, only 50 symbols out of 100 are plotted for the iterative method.

Perfect agreement is again obtained between the two solvers. Appearance of vapor in the liquid is computed without oscillations by the two methods. 


\section{Computational time, efficiency and simplicity}

All computational examples confsidered in this paper led to the same observation: the present relaxation solver is much faster than the root-finding iterative algorithm given in Appendix. In

our previous work [1] CPU savings from 5 to $50 \%$ were obtained, depending on the conditions. Due to the increased complexity of the iterative algorithm for the multi-component version, the CPU saving is now 50\% in all reported cases. The main argument for this time gain is related to its simplicity: the relaxation solver is direct whereas the iterative method requires solving a non-linear-algebraic system that may cause difficulties as a result of non-linearities. Besides, the root-finding iterative method requires the calculation of the saturation pressure via Eq. (2.4) at each iterative step, which itself requires an iterative method.

In practical applications, fine discretization is usually used on zones of interest. However, as the method is not iterative, and converges after some time steps, it is interesting to address its robustness and accuracy in situation with both coarse space and time resolutions. To this end, Fig 8 repeats the test case already presented in Fig 5, with a 10-cell mesh and first order Godunov numerical scheme. These results are very reasonable, illustrating the robustness of the method and its correct behavior even on coarse meshes. Results for the same test case are also given in Fig 8 for a higher-order solver (MUSCL-Hancock scheme with the Superbee limiter), on a 1000-cell mesh. Again, perfect agreement is found between the present relaxation solver and the non-linear iterative method. Robustness of the present method thus seems quite independent of mesh resolution and order of accuracy of the numerical scheme.

\section{Multi-dimensional illustrations}

\subsection{Evaporating liquid jet}

In this section, the capabilities of the flow model are illustrated on an evaporating liquid jet configuration in conditions typical of cryotechnic rocket engines during the ignition phase (for which the engine has not yet reached supercritical conditions). The flow consists of a coaxial liquid oxygen jet surrounded by a high-speed hydrogen flow, injected in conditions above the saturation point of the inner oxygen core, which then evaporates whilst being destabilized. Such a case is very challenging, because there is initially no vapor oxygen, and the phase transfer 

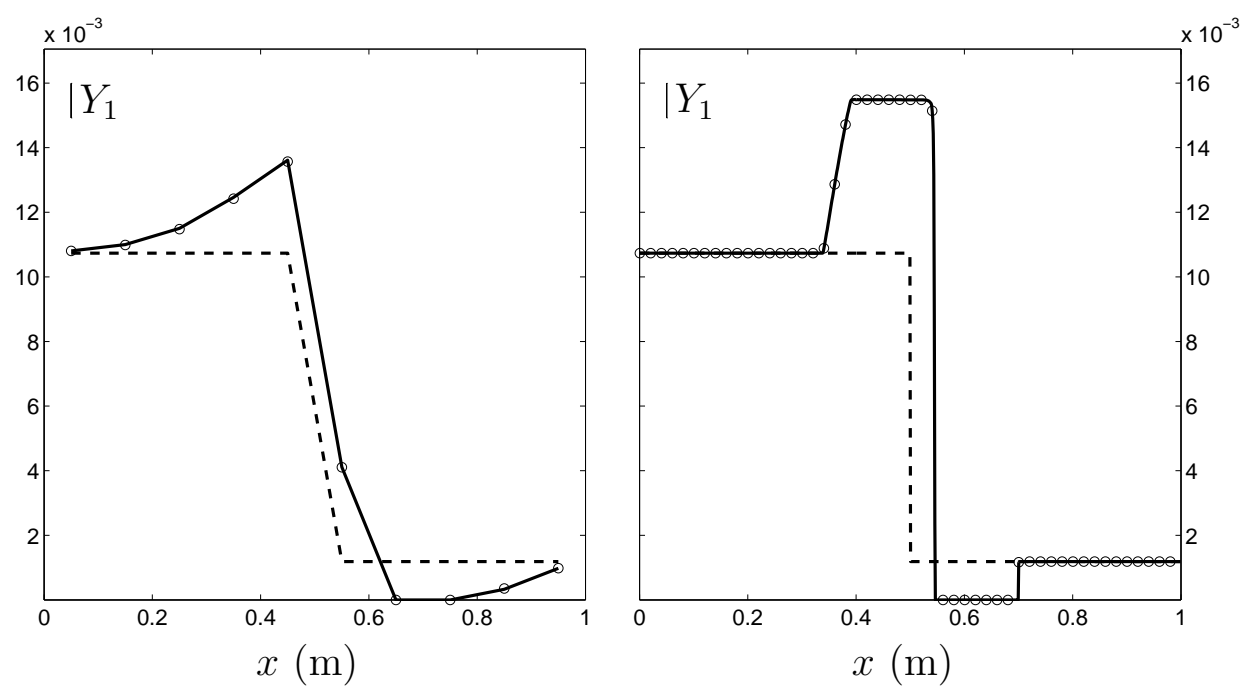

Figure 8: Comparison of the present relaxation algorithm (thick lines) versus the non-linear iterative solver (symbols). Shock tube test with a two-phase mixture mainly made of air, computed on coarse (left figure) and fine (right figure) meshes to assess method robustness. The dashed lines represent the initial conditions: $p_{l}=2 \cdot 10^{5} \mathrm{~Pa}, p_{r}=10^{5} \mathrm{~Pa}, u_{l}=u_{r}=0 \mathrm{~m} . \mathrm{s}^{-1}, Y_{1}^{\text {left }} \simeq 1.073 \cdot 10^{-2}, Y_{1}^{\text {right }} \simeq 1.186 \cdot 10^{-3}, Y_{2}^{\text {left }} \simeq 9.263 \cdot 10^{-3}$, $Y_{2}^{\text {right }} \simeq 1.881 \cdot 10^{-2}$ and $Y_{3}^{\text {left }}=Y_{3}^{\text {right }}=0.98$. Final time: $t \approx 0.5 \mathrm{~ms}$. The figure on the left represents the solutions with a 10-cell mesh and first order Godunov numerical scheme. The figure on the right represents the solutions with a 1000-cell mesh and MUSCL-Hancock scheme with Superbee limiter. For the sake of clarity, only 50 symbols out of 1000 are plotted for the iterative method in the figure on the right. The relaxation method and iterative one tend to the same solution even with the coarse mesh. 
model developed is the only possible term for vapor production. The Stiffened Gas EOS is used, the parameters for oxygen and hydrogen are given in Table 2 .

\begin{tabular}{|l|c|c|c|}
\hline Coefficients & Liquid phase $\left(\mathrm{O}_{2}\right)$ & Vapor phase $\left(\mathrm{O}_{2}\right)$ & Hydrogen $\left(\mathrm{H}_{2}\right)$ \\
\hline$C_{p}(\mathrm{~J} / \mathrm{kg} / \mathrm{K})$ & 1702 & 780 & 14256 \\
\hline$C_{v}(\mathrm{~J} / \mathrm{kg} / \mathrm{K})$ & 695 & 531 & 10183 \\
\hline$\gamma$ & 2.45 & 1.47 & 1.4 \\
\hline$P_{\infty}(\mathrm{bar})$ & 1062 & 0 & 0 \\
\hline$q(\mathrm{~J} / \mathrm{kg})$ & -258000 & 6900 & $-1.2 .10^{6}$ \\
\hline$q^{\prime}(\mathrm{J} / \mathrm{kg} / \mathrm{K})$ & 0 & -9280 & 0 \\
\hline
\end{tabular}

Table 2: Stiffened Gas coefficients for the simulation of the evaporating liquid jet.

The 2D computations are carried out on a simplified geometry given in Fig. 9,

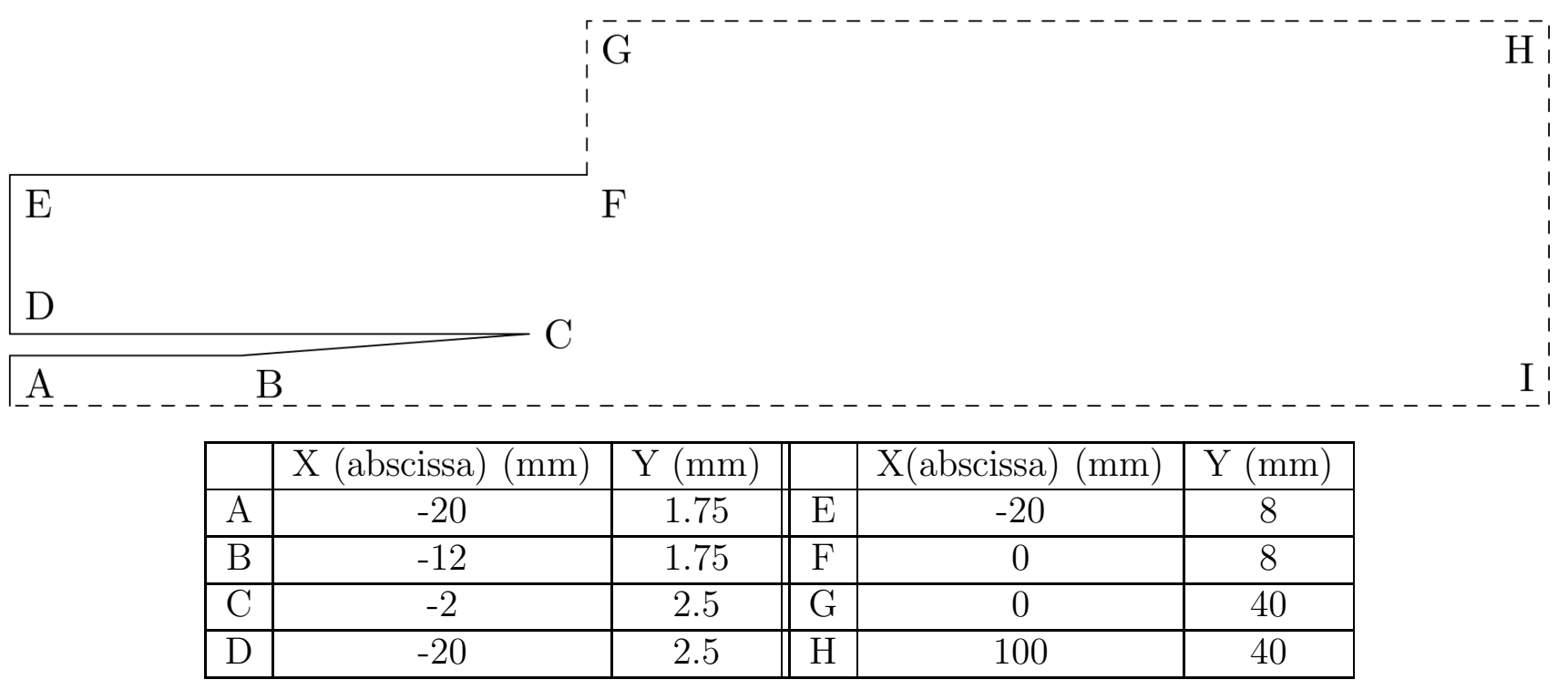

Figure 9: Geometrical data for half the computational domain of the evaporating liquid jet.

The inlet boundary conditions correspond to two subsonic inflows:

- central flow made of nearly pure liquid oxygen, at $100 \mathrm{~K}$ and $30 \mathrm{~m} \cdot \mathrm{s}^{-1}$, pressure $3 \mathrm{MPa}$ along segment AB of Fig. 9,

- peripheral flow made of nearly pure gaseous hydrogen, at $150 \mathrm{~K}$ and $200 \mathrm{~m} . \mathrm{s}^{-1}$, with the pressure of $3 \mathrm{MPa}$ along segment DE of Fig. 9, 
and one subsonic outlet, along segments GH and HI, where non-reflecting subsonic boundary condition is imposed at $3 \mathrm{MPa}$. The remaining walls are treated as symmetric boundary conditions. The mesh consists of 360, 000 triangles, with a spatial discretization varying from $0.1 \mathrm{~mm}$ in the jet to $0.6 \mathrm{~mm}$ in the far field.

The small scale destabilization of the liquid jet requires to extend the numerical solver presented in Saurel et al. (2016) [6] to second order. This was achieved following the MUSCLHancock scheme with the Superbee limiter, as detailed for example in Toro [14].

Two computations have been carried out on the same mesh, with the aim of studying the effect of the thermochemical relaxation on jet destabilization. The contours of mass fraction of liquid oxygen as obtained with and without evaporation effects are compared in Figs. 10, a and 10. b, showing serious changes due to phase transition.

As expected, the filaments separating the main liquid core and the gas gradually vanish as a consequence of evaporation, resulting in much steeper contours of liquid mass fraction in the vicinity of the jet, and very few pockets of liquid with significant life time. Fig. 10.d shows the contour of vapor oxygen (which indeed remains at zero in the case without phase transfer). Fig. 10.c plots the total oxygen mass fraction contour $\left(Y_{1}+Y_{2}\right)$. It can also be compared to Fig. 10, a

Although the results in terms of total mass fraction for oxygen qualitatively seem close whether or not the mass transfer is activated, it is clear that the vapor mass fraction is of utmost importance for our future work, which shall include the gaseous combustion between vapor oxygen and hydrogen.

Capillary effects are not included in these simulations, as the intense velocity gradients make them negligible in the present jet destabilization conditions. However, capillary effects may be of importance when the filaments start separating from the jet, and our future work will include them, following the approach presented in Le Martelot et al (2014) [15].

\subsection{Explosive liquid water dispersal into air}

The present relaxation solver has been presented in the context of the 4-equation formulation. This model considers mechanical and thermal equilibrium but thermochemical disequilibrium. However the relaxation solver is not restricted to such a model and can be extended to nonequilibrium hyperbolic flow models, such as Baer and Nunziato's (1986) [2] and its reduced 


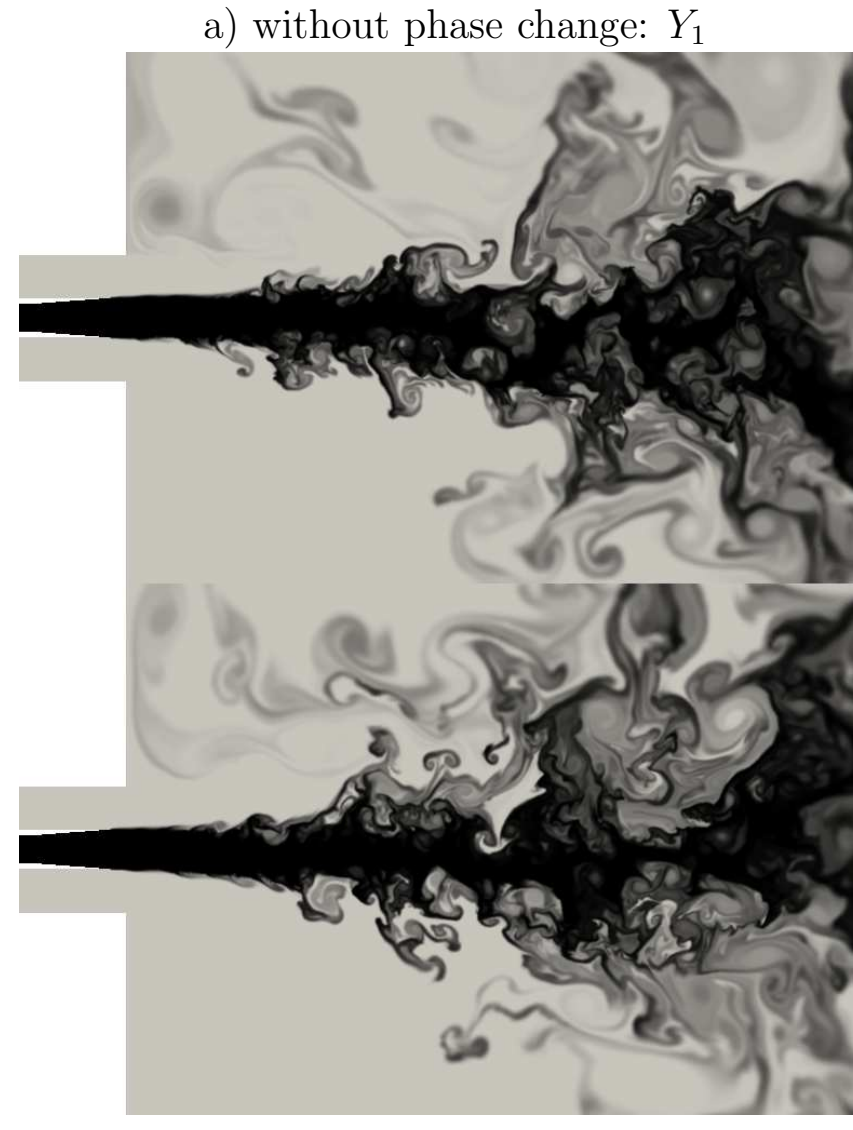

c) with phase change: $Y_{1}+Y_{2}$ b) with phase change: $Y_{1}$

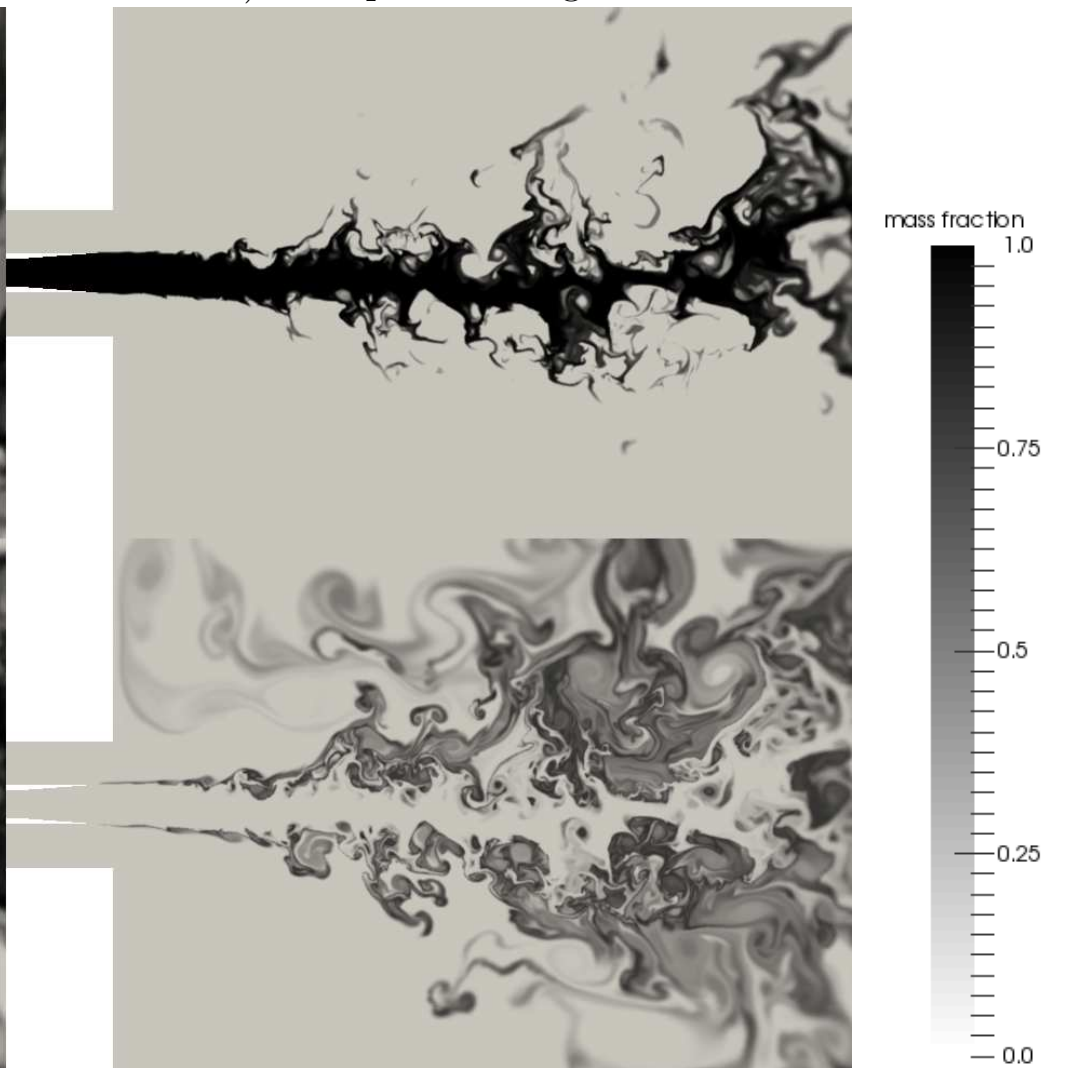

d) with phase change: $Y_{2}$

Figure 10: Fragmentation of a liquid $\mathrm{O}_{2}$ jet by a coaxial high-speed $\mathrm{H}_{2}$ current. The results from Figs b,c and $\mathrm{d}$ are from the same computation, whereas Fig a is taken at the same time, from a computation without phase transition. All results present mass fraction contours, on the same color map. Figs a) and b) compare the liquid mass fraction of oxygen without and with phase transfer. Fig. d) shows the contour of vapor oxygen resulting from the liquid jet evaporation. Fig. c) represents the sum $Y_{1}+Y_{2}($ Fig b $+\mathrm{c})$, showing a total mass fraction of oxygen, to be compared to the case without mass transfer (Fig. a). 
versions. This last model is a 7-equation formulation assuming mechanical, thermal and chemical disequilibrium. Thereby, finding local thermodynamic equilibrium requires extra ingredients such as velocity, pressure and temperature relaxation solvers such as the ones given in [9, 10]. We propose here to repeat the explosive liquid water dispersal test presented in Furfaro and Saurel (2015) [16] with the 4 and 7-equation models. The test consists in a cylindrical gas-liquid explosion schematically represented in Fig [11, The results are given in Fig 12 .

Nearly pure dense gas $\left(1500 \mathrm{~kg} / \mathrm{m}^{3}\right)$ HP chamber (7000 Mpa)

Nearly pure liquid water $\left(1000 \mathrm{~kg} / \mathrm{m}^{3}\right)$ Atmospheric pressure (0.1 Mpa)

Nearly pure gas $\left(1 \mathrm{~kg} / \mathrm{m}^{3}\right)$ Atmospheric pressure (0.1 Mpa)

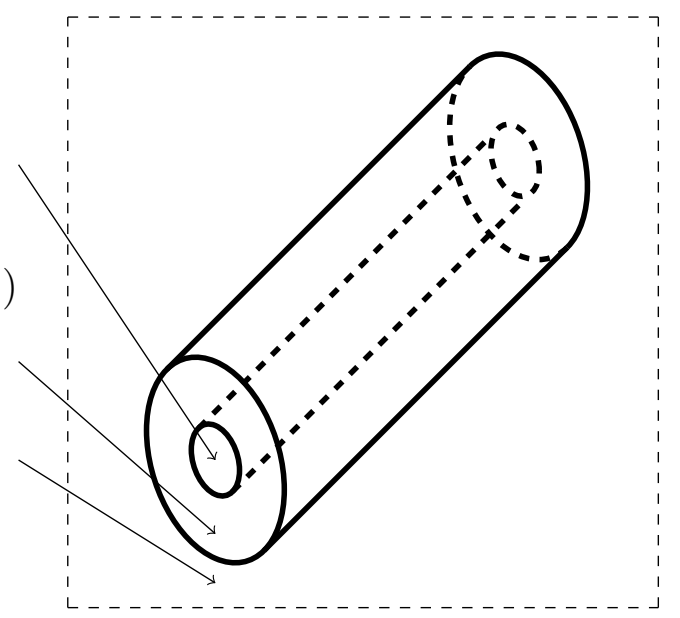

Figure 11: Schematic representation of the cylindrical gas-liquid explosive system. A cylindrical explosive charge is surrounded by a liquid water layer. The internal cylinder is $160 \mathrm{~mm}$ long with a $52 \mathrm{~mm}$ radius and is initially filled with nearly pure dense gas $\left(1500 \mathrm{~kg} / \mathrm{m}^{3}\right)$ at high pressure $(7000 \mathrm{Mpa})$. The external cylinder is $160 \mathrm{~mm}$ long with a $96 \mathrm{~mm}$ radius and is initially filled with nearly pure liquid water $\left(1000 \mathrm{~kg} / \mathrm{m}^{3}\right)$ at atmospheric pressure $(0.1 \mathrm{MPa})$. Atmospheric conditions with nearly pure gas $\left(1 \mathrm{~kg} / \mathrm{m}^{3}\right)$ at $0.1 \mathrm{MPa}$ are considered around both cylinders.

No noticeable changes are visible in Fig 12 illustrating the capabilities of the relaxation solver to model phase change in both 7-equation and 4-equation models . An evaporation front is clearly visible with both models.

\section{Conclusions}

A simple relaxation solver has been built, able to deal with phase transition between a liquid phase, and a multicomponent gas phase. Several features are in favor to the present solver compared to iterative ones:

- CPU savings over 50\%,

- high simplicity of implementation and verification, 

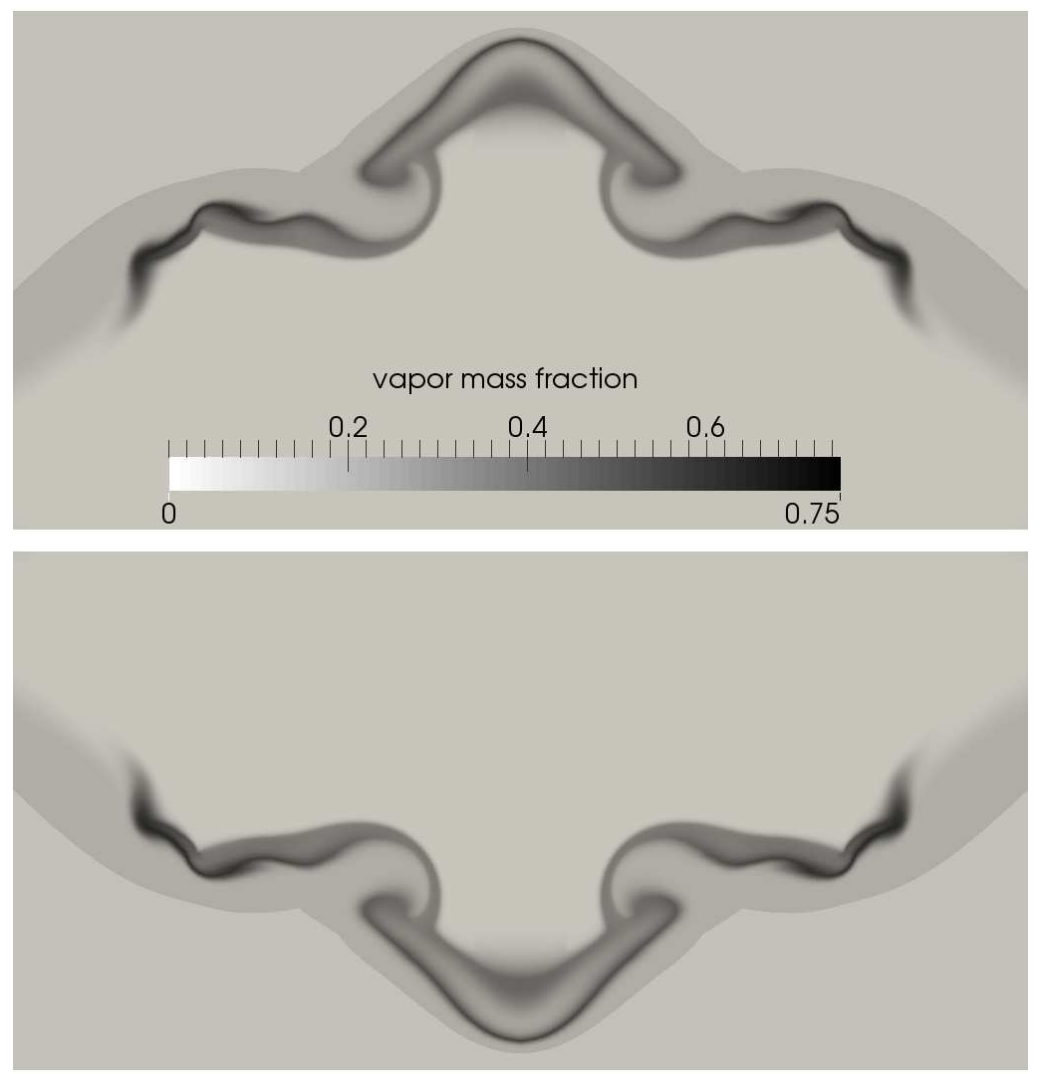

Figure 12: Vapor mass fraction created during a 2D cylindrical gas-liquid explosive dispersal. The figure compares the results obtained with the 4-equation model (mechanical and thermal equilibrium, top) and with the 7-equation model (total disequilibrium, bottom) after relaxation of the pressures, velocities, temperatures following the relaxations solvers presented in [9] and phase transition consideration through the relaxation method presented in this paper. The results computed by both models are in close agreement. 
- increased robustness with high-order methods.

This is reached without any noticeable loss of accuracy.

This research can be continued in many directions. Among them, consideration of supercritical fluids in the same theoretical frame appears important. Another perspective deals with the consideration of combustion effects within the gas phase.

\section{Acknowledgements}

Part of this work has been carried out in the framework of the Labex MEC (ANR-10LABX-0092) and of the A*MIDEX project (ANR-11-IDEX-0001-02), funded by the Investissements d'Avenir French Government program managed by the French National Research Agency (ANR). We also acknowledge funding from ANR through project ANR-14-CE22-0014.

Damien Furfaro and François Fraysse are gratefully acknowledged for carrying out the simulations presented in Fig. 12. 


\section{References}

[1] A. Chiapolino, P. Boivin, R. Saurel, A simple phase transition relaxation solver for liquidvapor flows, International Journal for Numerical Methods in Fluids 83 (7) (2017) 583-605.

[2] M. Baer, J. Nunziato, A two-phase mixture theory for the deflagration-to-detonation transition (DDT) in reactive granular materials, International Journal of Multiphase Flow 12 (6) (1986) 861-889.

[3] A. Kapila, R. Menikoff, J. Bdzil, S. Son, D. Stewart, Two-phase modeling of deflagrationto-detonation transition in granular materials: Reduced equations, Physics of Fluids 13 (10) (2001) 3002-3024.

[4] R. Saurel, F. Petitpas, R. Abgrall, Modelling phase transition in metastable liquids: application to cavitating and flashing flows, Journal of Fluid Mechanics 607 (2008) 313-350.

[5] S. LeMartelot, B. Nkonga, R. Saurel, Liquid and liquid-gas flows at all speeds, Journal of Computational Physics 255 (2013) 53-82.

[6] R. Saurel, P. Boivin, O. Le Métayer, A general formulation for cavitating, boiling and evaporating flows, Computers and Fluids 128 (2016) 53-64.

[7] P. Downar-Zapolski, Z. Bilicki, L. Bolle, J. Franco, The non-equilibrium relaxation model for one-dimensional flashing liquid flow, International Journal of Multiphase Flow 22 (3) (1996) 473-483.

[8] M. Barret, E. Faucher, J. Hérard, Schemes to compute unsteady flashing flows, AIAA Journal 40 (5) (2002) 905-913.

[9] O. Le Métayer, J. Massoni, R. Saurel, Dynamic relaxation processes in compressible multiphase flows. Application to evaporation phenomena, in: ESAIM: Proceedings, Vol. 40, EDP Sciences, 2013, pp. 103-123.

[10] M. Lallemand, R. Saurel, Pressure relaxation procedures for multiphase compressible flows, INRIA Report 403 (8). 
[11] O. Le Métayer, J. Massoni, R. Saurel, Elaborating equations of state of a liquid and its vapor for two-phase flow models; Elaboration des lois d'état d'un liquide et de sa vapeur pour les modèles d'écoulements diphasiques, International Journal of Thermal Sciences 43 (3) (2004) 265-276.

[12] O. Le Métayer, R. Saurel, The Noble-Abel Stiffened-Gas equation of state, Physics of Fluids 28 (4) (2016) 046102.

[13] D. Furfaro, R. Saurel, Modeling droplet phase change in the presence of a multi-component gas mixture, Applied Mathematics and Computation 272 (2016) 518-541.

[14] E. F. Toro, Riemann solvers and numerical methods for fluid dynamics: A practical introduction, Springer Science \& Business Media, 1997.

[15] S. Le Martelot, R. Saurel, B. Nkonga, Towards the direct numerical simulation of nucleate boiling flows, International Journal of Multiphase Flow 66 (2014) 62-78.

[16] D. Furfaro, R. Saurel, A simple HLLC-type Riemann solver for compressible non-equilibrium two-phase flows, Computers and Fluids 111 (2015) 159-178.

\section{AppendixA. Summary of the iterative algorithm}

The algebraic system to be solved is,

$$
\left\{\begin{array}{l}
v=Y_{1}^{*} v_{1}\left(p^{*}, T^{*}\right)+Y_{2}^{*} v_{2}\left(p^{*}, T^{*}\right)+\sum_{k \geqslant 3} Y_{k} v_{k}\left(p^{*}, T^{*}\right), \\
e=Y_{1}^{*} e_{1}\left(p^{*}, T^{*}\right)+Y_{2}^{*} e_{2}\left(p^{*}, T^{*}\right)+\sum_{k \geqslant 3} Y_{k} e_{k}\left(p^{*}, T^{*}\right), \\
p_{s a t}\left(T^{*}\right)=\left(\frac{\frac{Y_{2}^{*}}{W_{2}}}{\frac{Y_{2}^{*}}{W_{2}}+\sum_{k \geqslant 3} \frac{Y_{k}}{W_{k}}}\right) p^{*},
\end{array}\right.
$$


where the superscript ${ }^{*}$ denotes the thermodynamic equilibrium state. Since $Y_{1}=1-Y_{2}-$ $\sum_{k \geqslant 3} Y_{k}$, the algebraic system reads,

$$
\left\{\begin{array}{l}
f_{1}\left(p^{*}, T^{*}, Y_{2}^{*}\right)=v-\left(1-Y_{2}^{*}-\sum_{k \geqslant 3} Y_{k}\right) v_{1}\left(p^{*}, T^{*}\right)-Y_{2}^{*} v_{2}\left(p^{*}, T^{*}\right)-\sum_{k \geqslant 3} Y_{k} v_{k}\left(p^{*}, T^{*}\right), \\
f_{2}\left(p^{*}, T^{*}, Y_{2}^{*}\right)=e-\left(1-Y_{2}^{*}-\sum_{k \geqslant 3} Y_{k}\right) e_{1}\left(p^{*}, T^{*}\right)-Y_{2}^{*} e_{2}\left(p^{*}, T^{*}\right)-\sum_{k \geqslant 3} Y_{k} e_{k}\left(p^{*}, T^{*}\right), \\
f_{3}\left(p^{*}, p_{\text {sat }}^{*}, Y_{2}^{*}\right)=p_{\text {sat }}^{*}-\left(\frac{\frac{Y_{2}^{*}}{W_{2}}}{\frac{Y_{v}^{*}}{W_{2}}+\sum_{k \geqslant 3} \frac{Y_{k}}{W_{k}}}\right) p^{*} .
\end{array}\right.
$$

However the relation between $p_{\text {sat }}\left(T^{*}\right)$ and $T^{*}$,

$$
\ln \left(p_{\text {sat }}+p_{\infty, v}\right)=A+\frac{B+E p_{\text {sat }}}{T_{\text {sat }}}+C \ln \left(T_{\text {sat }}\right)+D \ln \left(p_{\text {sat }}+p_{\infty, l}\right),
$$

is non-linear and no analytical relation can be found. An additional equation is consequently added to System (A.2).

It is convenient to use a reference state defined with the other NASG or SG coefficients which have been determined via theoretical equations and experimental data (see Le Métayer et al. [11], [12]). Let us define,

$$
\left\{\begin{array}{l}
T_{r e f}=\frac{q_{1}-v_{2}}{C_{p, 2}-C_{v, 2}}=\frac{B}{C}, \\
p_{r e f}=\frac{\left(C_{p, 2}-C_{v, 2}\right) p_{\infty, 1}-\left(C_{p, 1}-C_{v, 1}\right) p_{\infty, 2}}{\left(C_{p, 1}-C_{v, 1}\right)-\left(C_{p, 2}-C_{v, 2}\right)}=\frac{p_{\infty, 1}-D p_{\infty, 2}}{D-1} .
\end{array}\right.
$$

Eq. (A.3) is now written with the built reference state $p_{r e f}, T_{r e f}$,

$$
\ln \left(p_{r e f}+p_{\infty, 2}\right)=A+\frac{B+E p_{r e f}}{T_{r e f}}+C \ln \left(T_{r e f}\right)+D \ln \left(p_{r e f}+p_{\infty, 1}\right)
$$


Subtracting Eq. (A.5) to Eq. (A.3), it is straightforward to find another formulation connecting the saturation pressure and temperature,

$$
\frac{p_{\text {sat }}+p_{\infty, 2}}{p_{\text {ref }}+p_{\infty, 2}}=\exp \left\{C\left[\frac{T_{r e f}}{T_{\text {sat }}}+\ln \left(\frac{T_{\text {sat }}}{T_{\text {ref }}}\right)-1\right]\right\}\left(\frac{p_{\text {sat }}+p_{\infty, 1}}{p_{\text {ref }}+p_{\infty, 1}}\right)^{D} \exp \left\{E\left(\frac{p_{\text {sat }}}{T_{\text {sat }}}-\frac{p_{\text {ref }}}{T_{\text {ref }}}\right)\right\} .
$$

The algebraic system thus reads,

$$
\left\{\begin{array}{l}
f_{1}\left(p^{*}, T^{*}, Y_{2}^{*}\right)=v-\left(1-Y_{2}^{*}-\sum_{k \geqslant 3} Y_{k}\right) v_{1}\left(p^{*}, T^{*}\right)-Y_{2}^{*} v_{2}\left(p^{*}, T^{*}\right)-\sum_{k \geqslant 3} Y_{k} v_{k}\left(p^{*}, T^{*}\right) \\
f_{2}\left(p^{*}, T^{*}, Y_{2}^{*}\right)=e-\left(1-Y_{2}^{*}-\sum_{k \geqslant 3} Y_{k}\right) e_{1}\left(p^{*}, T^{*}\right)-Y_{2}^{*} e_{2}\left(p^{*}, T^{*}\right)-\sum_{k \geqslant 3} Y_{k} e_{k}\left(p^{*}, T^{*}\right) \\
f_{3}\left(p^{*}, p_{\text {sat }}^{*}, Y_{2}^{*}\right)=p_{\text {sat }}^{*}-\left(\frac{\frac{Y_{2}^{*}}{W_{2}}}{\frac{Y_{2}^{*}}{W_{2}}+\sum_{k \geqslant 3} \frac{Y_{k}}{W_{k}}}\right) p^{*}, \\
f_{4}\left(p_{\text {sat }}^{*}, T^{*}\right)=\frac{p_{\text {sat }}^{*}+p_{\infty, 2}}{p_{\text {ref }}+p_{\infty, 2}}-\exp \left\{C\left[\frac{T_{r e f}}{T^{*}}+\ln \left(\frac{T^{*}}{T_{\text {ref }}}\right)-1\right]\right\}\left(\frac{p_{\text {sat }}^{*}+p_{\infty, 1}}{p_{\text {ref }}+p_{\infty, 1}}\right)^{D} \exp \left\{E\left(\frac{p_{\text {sat }}^{*}}{T^{*}}-\frac{p_{\text {ref }}}{T_{\text {ref }}}\right)\right\} .
\end{array}\right.
$$

Four variables are unknown, $p^{*}, T^{*}, Y_{v}^{*}, p_{s a t}^{*}$ and System (A.7) is non-linear calling for an iterative method such as Newton-Raphson's. Let us then denote,

$$
X=\left(\begin{array}{c}
p^{*} \\
T^{*} \\
Y_{v}^{*} \\
p_{\text {sat }}^{*}
\end{array}\right), \quad F(X)=\left(\begin{array}{c}
f_{1}\left(p^{*}, T^{*}, Y_{2}^{*}\right) \\
f_{2}\left(p^{*}, T^{*}, Y_{2}^{*}\right) \\
f_{3}\left(p^{*}, p_{s a t}^{*}, Y_{2}^{*}\right) \\
f_{4}\left(p_{\text {sat }}^{*}, T^{*}\right)
\end{array}\right) \text {. }
$$

The system $F(X)=0$ has to be resolved. Its solution is given by,

$$
X^{n}=X^{n-1}-J\left\{F\left(X^{n-1}\right)\right\}^{-1} F\left(X^{n-1}\right),
$$


with $n$ denoting the current iteration and $J\left\{F\left(X^{n-1}\right)\right\}^{-1}$ the inverse of the Jacobian matrix,

$$
J\left(f_{1}, f_{2}, f_{3}, f_{4}\right)=\left(\begin{array}{cccc}
\frac{\partial f_{1}\left(p^{*}, T^{*}, Y_{2}^{*}\right)}{\partial p^{*}} & \frac{\partial f_{1}\left(p^{*}, T^{*}, Y_{2}^{*}\right)}{\partial T^{*}} & \frac{\partial f_{1}\left(p^{*}, T^{*}, Y_{2}^{*}\right)}{\partial Y_{2}^{*}} & \frac{\partial f_{1}\left(p^{*}, T^{*}, Y_{2}^{*}\right)}{\partial p_{s a t}^{*}} \\
\frac{\partial f_{2}\left(p^{*}, T^{*}, Y_{2}^{*}\right)}{\partial p^{*}} & \frac{\partial f_{2}\left(p^{*}, T^{*}, Y_{2}^{*}\right)}{\partial T^{*}} & \frac{\partial f_{2}\left(p^{*}, T^{*}, Y_{2}^{*}\right)}{\partial Y_{2}^{*}} & \frac{\partial f_{2}\left(p^{*}, T^{*}, Y_{2}^{*}\right)}{\partial p_{s a t}^{*}} \\
\frac{\partial f_{3}\left(p^{*}, p_{s a t}^{*}, Y_{2}^{*}\right)}{\partial p^{*}} & \frac{\partial f_{3}\left(p^{*}, p_{s a t}^{*}, Y_{2}^{*}\right)}{\partial T^{*}} & \frac{\partial f_{3}\left(p^{*}, p_{s a t}^{*}, Y_{2}^{*}\right)}{\partial Y_{2}^{*}} & \frac{\partial f_{3}\left(p^{*}, p_{s a t}^{*}, Y_{2}^{*}\right)}{\partial p_{s a t}^{*}} \\
\frac{\partial f_{4}\left(p_{s a t}^{*}, T^{*}\right)}{\partial p^{*}} & \frac{\partial f_{4}\left(p_{s a t}^{*}, T^{*}\right)}{\partial T^{*}} & \frac{\partial f_{4}\left(p_{s a t}^{*}, T^{*}\right)}{\partial Y_{v}^{*}} & \frac{\partial f_{4}\left(p_{s a t}^{*}, T^{*}\right)}{\partial p_{s a t}^{*}}
\end{array}\right)
$$

In order to gain accuracy and robustness, it is convenient to write Eq. (A.9) as,

$$
\left\{\begin{aligned}
J\left(F\left(X^{n-1}\right)\right) \triangle X & \left.=-F\left(X^{n-1}\right)\right), \\
\triangle X & =X^{n}-X^{n-1} .
\end{aligned}\right.
$$

Thereby, for a given $X$, a linear system is found and the variation $\triangle X$ is determined via the Gauss elimination method. The variables are then updated as,

$$
X^{n}=\triangle X+X^{n-1}
$$

A solution is obtained when $\triangle X<\epsilon$ or alternatively when $\left|f_{1}\right|<\epsilon,\left|f_{2}\right|<\epsilon,\left|f_{3}\right|<\epsilon,\left|f_{4}\right|<\epsilon$, with $\epsilon \rightarrow 0$.

This procedure is robust and accurate. It is used in all test problems of Section 6. 